\title{
Scalable Constant pH Molecular Dynamics in GROMACS
}

\author{
Noora Aho, ${ }^{*, \dagger}$ Pavel Buslaev, ${ }^{*} \dagger$ Anton Jansen ${ }^{\ddagger}$ Paul Bauer, ${ }^{\ddagger}$ Gerrit Groenhof, ${ }^{*}, \dagger$ \\ and Berk Hess*, \\ $\dagger$ Nanoscience Center and Department of Chemistry, University of Jyväskylä, Finland \\ $\ddagger$ Department of Applied Physics, Science for Life Laboratory, KTH Royal Institute of \\ Technology, Stockholm, Sweden
}

E-mail: noora.s.aho@jyu.fi; pavel.i.buslaev@jyu.fi; gerrit.x.groenhof@jyu.fi; berk.hess@scilifelab.se

\begin{abstract}
Molecular dynamics (MD) computer simulations are used routinely to compute atomistic trajectories of complex systems. Systems are simulated in various ensembles, depending on the experimental conditions one aims to mimic. While constant energy, temperature, volume, and pressure are rather straightforward to model, $\mathrm{pH}$, which is an equally important parameter in experiments, is more difficult to account for in simulations. Although a constant $\mathrm{pH}$ algorithm based on the $\lambda$-dynamics approach by Brooks and co-workers was implemented in a fork of the GROMACS molecular dynamics program, uptake has been rather limited, presumably due to the poor scaling of that code with respect to the number of titratable sites. To overcome this limitation, we implemented an alternative scheme for interpolating the Hamiltonians of the protonation states that makes the constant $\mathrm{pH}$ molecular dynamics simulations almost as fast as a normal MD simulation with GROMACS. In addition, we implemented a simpler scheme, called multisite representation, for modeling side chains with multiple titratable sites, such as imidazole rings. This scheme, which is based on constraining the
\end{abstract}


sum of the $\lambda$-coordinates, not only reduces the complexity associated with parameterizing the intra-molecular interactions between the sites, but is also easily extendable to other molecules with multiple titratable sites. With the combination of a more efficient interpolation scheme and multisite representation of titratable groups, we anticipate a rapid uptake of constant $\mathrm{pH}$ molecular dynamics simulations within the GROMACS user community.

\section{Introduction}

Since their introduction more than four decades ago, molecular dynamics (MD) computer simulations have come of age. ${ }^{1}$ Thanks to improvements in computer hardware, algorithmic developments, as well as increased accuracy of force fields, MD simulation has evolved into a predictive technique that can complement experiment by providing atomistic insights into the dynamics of complex systems. ${ }^{1,2}$ While many experimental conditions can be modelled with good accuracy, the aqueous proton concentration, or $\mathrm{pH}$, is typically accounted for indirectly by constraining the protonation states of titratable residues to their, presumed, most probable form at the start of the simulation. Because the electrostatic interactions depend critically on the protonation state of the residues, the $\mathrm{pH}$ affects the conformational ensemble. Conversely, because the conformation can influence the proton affinity of the residues, or $\mathrm{p} K_{\mathrm{a}}$, a direct correlation exists between $\mathrm{pH}$ and conformational dynamics, which cannot be captured if protonation state is kept fixed in the simulation. ${ }^{3}$

To overcome this limitation in classical MD simulations and include the effect of $\mathrm{pH}$ on the conformational sampling directly, several solutions have been proposed in the last

decades. ${ }^{4,5}$ These solutions can be roughly divided into a category that relies on discrete changes in protonation states, ${ }^{6-12}$ and a second category, in which a protonation state can change continuously. ${ }^{13-25}$ More recently, a third category that relies on the transfer of protonlike particles between titratable sites, including protein residues and solvent molecules, was proposed for the Martini force field. ${ }^{26}$ 
In the discrete constant $\mathrm{pH}$ approaches, the protonation state of a residue can change at regular intervals of the simulation according to a Metropolis Monte Carlo criterion. ${ }^{10,11,21,27,28}$ To avoid a low acceptance rate due to unfavourable solvent configurations, the Monte Carlo step is performed based on free energies calculated using the approximation of either an implicit solvent representation, ${ }^{7,9}$ or a short all-atom thermodynamic integration. ${ }^{8,29}$

Most continuous approaches for MD at constant $\mathrm{pH}$ are based on the $\lambda$-dynamics technique developed by Brooks and co-workers. ${ }^{30} \mathrm{~A}$ one-dimensional $\lambda$-coordinate with fictitious mass $m_{\lambda}$ is introduced for each titratable site, and the equations of motion for these additional degrees of freedom are integrated along with the Cartesian positions of the atoms. ${ }^{14}$ The $\lambda$-coordinate defines the protonation state of the residue: at $\lambda=0$ the residue is protonated and interacts with the rest of the system as such, while at $\lambda=1$ it is deprotonated. The energy function that acts on the $\lambda$-coordinates depends on (i) the intrinsic proton affinity (reference $\mathrm{p} K_{\mathrm{a}}$ ) of the titratable site in water, (ii) the interactions with the environment, which are mostly electrostatic, ${ }^{31}$ and (iii) the $\mathrm{pH}$ of the solvent, which is set by the user. In addition, potentials are introduced to bias sampling towards the physical states at $\lambda=0$ and $\lambda=1$. Protons are not transferred directly between the titratable residues and the solvent molecules, but rather exchanged with an external proton bath. Because the chemical potential of this bath is determined by the proton concentration $(\mathrm{pH})$ of the aqueous solution, constant pH MD (CpHMD) simulations based on $\lambda$-dynamics are performed in a grand canonical ensemble for the proton degrees of freedom.

While $\lambda$-dynamics based constant $\mathrm{pH}$ approaches were originally developed for implicit solvent simulations, ${ }^{14}$ they have since then been adapted for explicit solvent simulations as well. ${ }^{12,16-19,21,23}$ The key computational challenge for explicit solvent implementations is the long-range electrostatic interaction, for which multiple solutions have been suggested, including a shifted cut-off scheme, ${ }^{19}$ a hybrid scheme combining the particle mesh Ewald (PME) treatment for the Cartesian coordinates with the generalized Born model for the $\lambda$ particles, ${ }^{17,32}$ and a fully consistent PME treatment for both $\lambda$ and Cartesian degrees of 
freedom. ${ }^{16,23}$

In addition to an accurate modeling of the long-range electrostatic interactions, also sampling can pose a serious challenge to simulations at constant $\mathrm{pH}$. While the choice for the PME method in the original implementation of $\lambda$-dynamics in the fork of GROMACS 3.3 release ${ }^{16}$ was motivated by its accurate description of long-range electrostatics, the linear increase of the computational effort with the number of titratable sites limited the sampling efficiency, which meant that systems with many titratable sites could not be studied in practice.

To remove this bottleneck and enable constant $\mathrm{pH}$ MD with GROMACS at modest extra cost compared to a standard simulation, we switch to an alternative scheme for computing the long-range electrostatic interactions of the $\lambda$-particles under periodic boundary conditions. The alternative scheme is based on a linear interpolation of partial charges, ${ }^{14}$ rather than the potential energy functions as in the original implementation of constant $\mathrm{pH} \mathrm{MD}$ in a GROMACS fork. ${ }^{16}$

Although the previous implementation of constant $\mathrm{pH}$ in a GROMACS fork was documented and shared with the community as an open-source program, there has been some misunderstanding about how electrostatic interactions were computed for $\lambda$-particles. ${ }^{17,18,23}$ To resolve this, we first explain in detail how the electrostatic interactions were calculated in the previous GROMACS implementation of constant pH MD. We next contrast this linear interpolation between the potential energy functions of the protonated and deprotonated states of a residue, with the interpolation between the partial charges of both states, ${ }^{14}$ and show why the latter is computationally much more efficient. We then demonstrate the superior performance of the charge-interpolation scheme by running a series of constant $\mathrm{pH}$ MD simulations of amino acids and proteins. To emphasize that the new constant $\mathrm{pH}$ implementation in GROMACS is not linked to a specific force field or method for evaluating electrostatic interactions, we also show the results of constant $\mathrm{pH}$ MD simulations with the Martini coarse-grained force field. ${ }^{33}$ Because of GROMACS' large user community, we expect 
our work to increase popularity of constant $\mathrm{pH}$ MD simulations.

\section{Theory}

Before discussing the differences between linear interpolating of the potential energy functions on the one hand, ${ }^{16}$ and of partial charges on the other hand, ${ }^{14}$ for computing the potential energy landscape of the titration coordinates, we briefly review the $\lambda$-dynamics approach $^{30}$ that forms the basis for the constant $\mathrm{pH}$ molecular dynamics algorithm in GROMACS. ${ }^{16}$

\section{$\lambda$-dynamics based constant $\mathrm{pH}$ MD simulations}

A titratable site $i$ can exist in a protonated or deprotonated state. The protonation state affects the interactions between the site and the rest of the system. In constant $\mathrm{pH}$ MD simulations based on $\lambda$-dynamics, ${ }^{30}$ an additional coordinate $\lambda_{i}$ is introduced for each site $i$, and the potential energy function of the total system is continuously interpolated between the two protonation states along this coordinate i.e., $V\left(\lambda_{i}\right) .{ }^{14}$ A fictitious mass $m_{\lambda}$ is assigned to each $\lambda_{i}$-coordinate, and the coordinates evolve along with the Cartesian degrees of freedom of all atoms in the system, based on Newton's equations of motion. Thus, the total Hamiltonian of the system is

$$
H(\mathbf{R}, \boldsymbol{\lambda})=\sum_{i}^{N_{\text {sites }}} \frac{m_{\lambda}}{2} \dot{\lambda}_{i}^{2}+\sum_{j}^{N_{\text {atoms }}} \frac{m_{j}}{2} \dot{\mathbf{r}}_{j}^{2}+V(\mathbf{R}, \boldsymbol{\lambda})
$$

where $\mathbf{R}$ is the vector of the Cartesian coordinates $\mathbf{r}_{j}$ of all $N_{\text {atoms }}$ atoms with mass $m_{j}$, and $\boldsymbol{\lambda}$ is the vector of the $\lambda_{i}$ coordinates of all $N_{\text {sites }}$ titratable sites.

\section{$\lambda$-dependent potential energy function}

In addition to the interpolation between the potentials of the protonated $V_{A}(\mathbf{R})$ and depro-

tonated states $V_{B}(\mathbf{R})$, three more $\lambda$-dependent terms are included in the potential energy 
function of the total system $V(\mathbf{R}, \boldsymbol{\lambda})$, as illustrated in Figure 1: (i) a correction potential $V_{i}^{\mathrm{MM}}\left(\lambda_{i}\right)$ to compensate for missing quantum mechanical contributions to proton affinities (Figure 1B); (ii) a biasing potential $V_{i}^{\text {bias }}\left(\lambda_{i}\right)$ that enhances sampling of the physical end states at $\lambda_{i}=0$ and $\lambda_{i}=1$ (Figure $1 \mathrm{C}$ ); and (iii) a pH-dependent term $V^{\mathrm{pH}}\left(\lambda_{i}\right)$ to model the chemical potential of protons in water (Figure 1D).

The purpose of adding the correction term $V_{i}^{\mathrm{MM}}\left(\lambda_{i}\right)$ (Figure 1B) is to make the interpolated potential function flat if the titratable site $i$ is in its reference state, for which the proton affinity is known experimentally, at $\mathrm{pH}=\mathrm{p} K_{\mathrm{a}, i}$. This potential is determined by evaluating the deprotonation free energy of the single residue in water (reference state) at the force field level by thermodynamic integration along the $\lambda$-coordinate (Figure $1 \mathrm{~A}$ ):

$$
V_{i}^{\mathrm{MM}}\left(\lambda_{i}\right)=-\Delta G_{i}^{\mathrm{MM}}\left(\lambda_{i}\right)
$$

To prevent sampling of the non-physical states between $\lambda_{i}=0$ and $\lambda_{i}=1$ on this flat potential energy surface while still enabling sufficient transitions between the physical end-states to sample both protonation states with the correct thermodynamic weight, we introduce the biasing potential $V_{i}^{\text {bias }}\left(\lambda_{i}\right)$ suggested by Donnini et al. ${ }^{34}$ (Figure $1 \mathrm{C}$ ).

The $\mathrm{pH}$ dependent term $V^{\mathrm{pH}}(\lambda)$ (Figure 1D) is a correction to include the effect of the solution $\mathrm{pH}$ on the free energy difference between the protonated and deprotonated states, such that this difference is

$$
V^{\mathrm{pH}}\left(\lambda_{i}=1\right)-V^{\mathrm{pH}}\left(\lambda_{i}=0\right)=R T \ln (10)\left[\mathrm{p} K_{\mathrm{a}, i}-\mathrm{pH}\right]
$$

where we use the experimentally determined $\mathrm{p} K_{\mathrm{a}, i}$ values of residue $i$ in its reference state. Although various forms for this potential have been suggested, ${ }^{22,34,35}$ we propose a smooth 
step-function-based potential:

$$
\begin{aligned}
& V^{\mathrm{pH}}\left(\lambda_{i}\right)=R T \ln (10)\left[\mathrm{p} K_{\mathrm{a}, i}-\mathrm{pH}\right] \frac{1}{1+\exp \left(-2 k_{1}\left(\lambda_{i}-1+x_{0}\right)\right.}, \text { if } \mathrm{pH}>\mathrm{p} K_{\mathrm{a}} \\
& V^{\mathrm{pH}}\left(\lambda_{i}\right)=R T \ln (10)\left[\mathrm{p} K_{\mathrm{a}, i}-\mathrm{pH}\right] \frac{1}{1+\exp \left(-2 k_{1}\left(\lambda_{i}-x_{0}\right)\right.}, \text { if } \mathrm{pH} \leq \mathrm{p} K_{\mathrm{a}},
\end{aligned}
$$

where $k_{1}$ and $x_{0}$ define the steepness and kink position of the step function. In this form, illustrated in Figure 1D, the $\mathrm{pH}$ dependent potential also aids in preventing the sampling of non-physical states, $i$. e., $0.1<\lambda<0.9$.
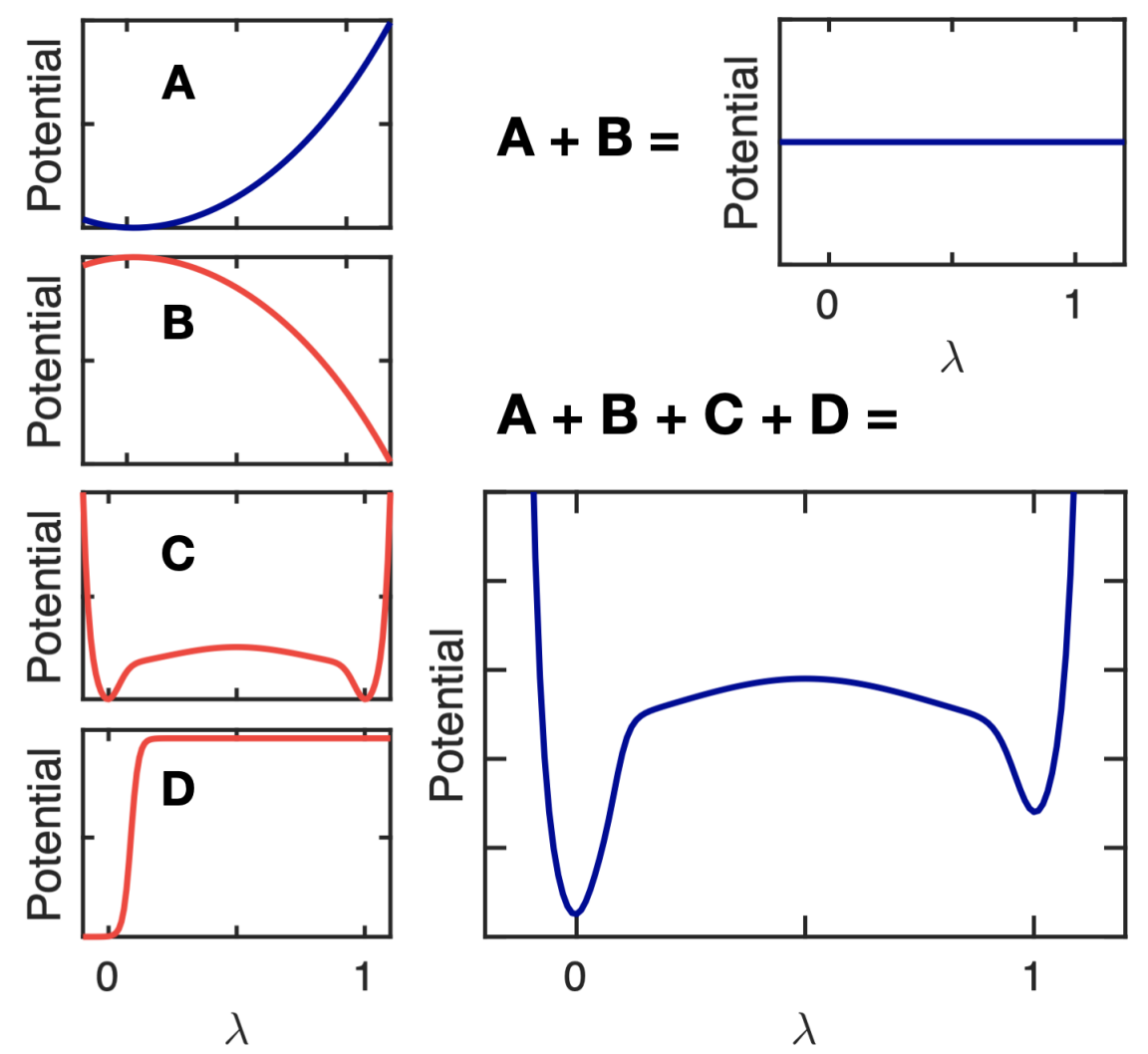

$A+B+C+D=$

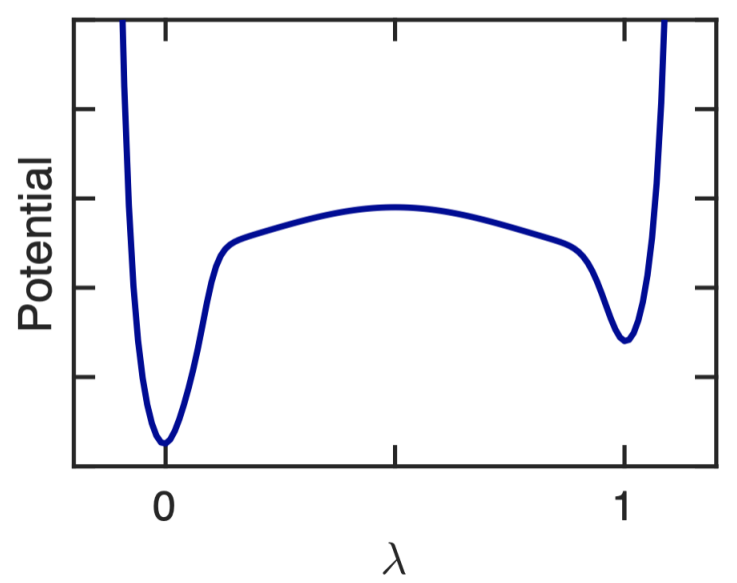

Figure 1: Illustration of the additional $\lambda$-dependent potential energy terms (B-D). Panel A shows the protonation free energy of a titratable residue in its reference state obtained at the force field level, $\Delta G_{i}^{\mathrm{MM}}(\lambda)$. To compensate for the shortcomings of the force field and obtain a zero free energy difference between the two protonation states $(\mathrm{A}+\mathrm{B})$, we add the correction potential, $V_{i}^{\mathrm{MM}}(\lambda)$, shown in panel B. A biasing potential, $V^{\text {bias }}(\lambda),{ }^{34}$ is introduced to avoid sampling of non-physical states (panel $\mathrm{C}$ ). To model the proton chemical potential $(\mathrm{pH})$, we add a $\mathrm{pH}$ dependent term, $V^{\mathrm{pH}}\left(\lambda_{i}\right)$ (panel D). For a titratable residue at $\mathrm{pH} \neq \mathrm{p} K_{\mathrm{a}}$, the total $\lambda$-dependent potential, including the interpolated force field functions and the three additional terms, is illustrated in panel $(\mathrm{A}+\mathrm{B}+\mathrm{C}+\mathrm{D})$. 


\section{Linear interpolation of potential energy functions}

In the previous implementation of constant $\mathrm{pH}$ MD in GROMACS' fork, the smooth interpolation of the force field potential energy function between the protonated and deprotonated states was achieved by linearly interpolating the force field potentials of these states. ${ }^{16}$ Thus, for a single titratable site, the $\lambda$-dependent potential is given by

$$
V(\mathbf{R}, \lambda)=(1-\lambda) V_{A}(\mathbf{R})+\lambda V_{B}(\mathbf{R})+V^{\mathrm{MM}}(\lambda)+V^{\text {bias }}(\lambda)+V^{\mathrm{pH}}(\lambda)
$$

with $V^{\mathrm{MM}}(\mathbf{R}, \lambda), V^{\text {bias }}(\lambda)$ and $V^{\mathrm{pH}}(\lambda)$ the correction, biasing, and $\mathrm{pH}$ dependent potentials, respectively, that were briefly discussed above, and with a short-hand notations for

$$
\begin{aligned}
& V_{A}(\mathbf{R})=V(\mathbf{R}, \lambda=0) \\
& V_{B}(\mathbf{R})=V(\mathbf{R}, \lambda=1)
\end{aligned}
$$

The gradient required for updating $\lambda$ according to Newton's equations of motion is

$$
\frac{\partial V(\mathbf{R}, \lambda)}{\partial \lambda}=V_{B}(\mathbf{R})-V_{A}(\mathbf{R})+\frac{\partial}{\partial \lambda} V^{\mathrm{MM}}(\mathbf{R}, \lambda)+\frac{\partial}{\partial \lambda} V^{\text {bias }}(\lambda)+\frac{\partial}{\partial \lambda} V^{\mathrm{pH}}(\lambda)
$$

Thus, the evaluation of the force on the $\lambda$-particle requires that the potential energy, including the long-range electrostatic interactions, is computed twice: once for $\lambda=0$ (i.e., $V_{A}$ ) and once more for $\lambda=1$ (i.e. $V_{B}$ ). If the Particle-Mesh-Ewald (PME) method is used to compute those long-range electrostatic interactions, ${ }^{36,37}$ separate PME grid builds are needed, because the charge distributions are not identical in states $A$ and $B$.

For systems with many titratable sites, multiple $\lambda$-groups are introduced. Because the analytical expressions for the correction, biasing and $\mathrm{pH}$ dependent terms in equation 5 are additive, we no longer consider them explicitly in what follows and focus exclusively on the interpolation of the force field potential energies between the multiple protonation states of the system. For $N \lambda$-coordinates, there are $2^{N}$ such states and the interpolation generalizes 
to: ${ }^{13}$

$$
V(\mathbf{R}, \boldsymbol{\lambda})=\sum_{\mathbf{l}}^{2^{N}} V(\mathbf{R}, \mathbf{l}) \Pi_{i}^{N} \lambda_{i}^{\left(l_{i}\right)}\left(1-\lambda_{i}\right)^{1-l_{i}}
$$

Here, we represent the $N \lambda_{i}$-coordinates as an $N$-dimensional vector $\boldsymbol{\lambda}$. The $2^{N}$ possible protonation states of the system are represented by the $N$-dimensional vector $\mathbf{l}$ with elements $l_{i}$ equal to 0 or 1 that indicate whether a site $i$ is protonated $\left(\lambda_{i}=0\right)$ or deprotonated $\left(\lambda_{i}=1\right)$. The sum runs over all $2^{N}$ possible combinations of $l_{i}=0$ and $l_{i}=1$. The gradient required for updating $\lambda_{i}$ is obtained by deriving the interpolated potential, $V(\mathbf{R}, \boldsymbol{\lambda})$, with respect to $\lambda_{i}:$

$$
\begin{aligned}
\frac{\partial}{\partial \lambda_{i}} V(\mathbf{R}, \boldsymbol{\lambda})= & \frac{\partial}{\partial \lambda_{i}}\left[\sum_{\mathbf{l}}^{2^{N}} V(\mathbf{R}, \mathbf{l}) \Pi_{k}^{N} \lambda_{k}^{\left(l_{k}\right)}\left(1-\lambda_{k}\right)^{1-l_{k}}\right] \\
= & \frac{\partial}{\partial \lambda_{i}}\left[\sum_{\mathbf{l}^{\prime}, \mathbf{l}_{\mathbf{i}}=\mathbf{0}}^{2^{N-1}} V(\mathbf{R}, \mathbf{l}) \Pi_{k}^{N} \lambda_{k}^{\left(l_{k}\right)}\left(1-\lambda_{k}\right)^{1-l_{k}}\left(1-\lambda_{i}\right)\right. \\
& \left.+\sum_{\mathbf{l}^{\prime}, l_{i}=1}^{2^{N-1}} V(\mathbf{R}, \mathbf{l}) \Pi_{k}^{N} \lambda_{k}^{\left(l_{k}\right)}\left(1-\lambda_{k}\right)^{1-l_{k}} \lambda_{i}\right] \\
= & \sum_{\mathbf{l}^{\prime}, l_{i}=1}^{2^{N-1}} V(\mathbf{R}, \mathbf{l}) \Pi_{k}^{N} \lambda_{k}^{\left(l_{k}\right)}\left(1-\lambda_{k}\right)^{1-l_{k}} \\
& -\sum_{\mathbf{l}^{\prime}, l_{i}=0}^{2^{N-1}} V\left(\mathbf{R}, \mathbf{l}^{\prime}\right) \Pi_{k}^{N} \lambda_{k}^{\left(l_{k}\right)}\left(1-\lambda_{k}\right)^{1-l_{k}} \\
= & V\left(\mathbf{R}, \boldsymbol{\lambda}^{\prime}, \lambda_{i}=1\right)-V\left(\mathbf{R}, \boldsymbol{\lambda}^{\prime}, \lambda_{i}=0\right)
\end{aligned}
$$

where the ${ }^{\prime}$ indicates that $\lambda_{i}$ is omitted from vector $\boldsymbol{\lambda}$. Note that, as we focus only on the interpolated potentials, the biasing, correction and $\mathrm{pH}$-dependent terms are left out.

In general, the number of terms in the potential (Equation 7) increases exponentially with the number of titratable sites. However, for pair-wise interactions involving titratable sites whose non-bonded force field parameters do not depend on the protonation state of the other sites (chemically uncoupled sites), the number of terms required to evaluate the interpolated potential scales linearly. For systems with such "chemically", or "topologically" 
uncoupled sites, the interpolated potential contains four types of interactions

$$
V(\mathbf{R}, \boldsymbol{\lambda})=V^{\text {rest-rest }}(\mathbf{R})+V^{\boldsymbol{\lambda} \text {-rest }}(\mathbf{R}, \boldsymbol{\lambda})+V^{\boldsymbol{\lambda}-\boldsymbol{\lambda}}(\mathbf{R}, \boldsymbol{\lambda})+V^{\boldsymbol{\lambda}}(\mathbf{R}, \boldsymbol{\lambda})
$$

For pair-wise electrostatic interactions, the terms on the right-hand side are defined as:

1. Interactions between atoms that are not part of any $\lambda$-group, and hence independent of the $\lambda_{i}$ 's:

$$
V^{\text {rest-rest }}(\mathbf{R})=\frac{1}{2} \sum_{i}^{n_{\text {rest }}} \sum_{j}^{n_{\text {rest }}} \frac{q_{i} q_{j}}{4 \pi \epsilon_{0} r_{i j}}
$$

where the sums run over all $n_{\text {rest }}$ atoms that are not part of a $\lambda$ group.

2. Interpolated interactions between atoms of each $\lambda$-group with atoms that are not part of any $\lambda$-group:

$$
V^{\boldsymbol{\lambda} \text {-rest }}(\mathbf{R}, \boldsymbol{\lambda})=\sum_{k}^{N_{\text {sites }}} \sum_{i}^{n_{k}} \sum_{j}^{n_{\text {rest }}}\left(1-\lambda_{k}\right) \frac{q_{i}^{A} q_{j}}{4 \pi \epsilon_{0} r_{i j}}+\lambda_{k} \frac{q_{i}^{B} q_{j}}{4 \pi \epsilon_{0} r_{i j}}
$$

where the first sum runs over all titratable sites, the second one runs over all $n_{k}$ atoms of the $k$-th $\lambda$-group and final sum runs over all atoms that are not part of any $\lambda$ group.

3. Interpolated interactions between atoms belonging to two different $\lambda$-groups:

$$
\begin{aligned}
V^{\boldsymbol{\lambda}-\boldsymbol{\lambda}}(\mathbf{R}, \boldsymbol{\lambda})= & \sum_{k}^{N_{\text {sites }}}\left[\left(1-\lambda_{k}\right) \sum_{m, m \neq k}^{N_{\text {sites }}} \sum_{i}^{n_{k}} \sum_{j}^{n_{m}}\left[\left(1-\lambda_{m}\right) \frac{q_{i}^{A} q_{j}^{A}}{4 \pi \epsilon_{0} r_{i j}}+\lambda_{m} \frac{q_{i}^{A} q_{j}^{B}}{4 \pi \epsilon_{0} r_{i j}}\right]\right. \\
& \left.\quad+\lambda_{k} \sum_{m, m \neq k}^{N_{\text {sites }}} \sum_{i}^{n_{k}} \sum_{j}^{n_{m}}\left[\left(1-\lambda_{m}\right) \frac{q_{i}^{B} q_{j}^{A}}{4 \pi \epsilon_{0} r_{i j}}+\lambda_{m} \frac{q_{i}^{B} q_{j}^{B}}{4 \pi \epsilon_{0} r_{i j}}\right]\right] \\
= & \sum_{k}^{N_{\text {sites }}} \sum_{m, m \neq k}^{N_{\text {sites }}} \sum_{i}^{n_{k}} \sum_{j}^{n_{m}} \frac{\left[\left(1-\lambda_{k}\right) q_{i}^{A}+\lambda_{k} q_{i}^{B}\right]\left[\left(1-\lambda_{m}\right) q_{j}^{A}+\lambda_{m} q_{j}^{B}\right]}{4 \pi \epsilon_{0} r_{i j}}
\end{aligned}
$$


4. Interpolated interactions within each of the $\lambda$-groups:

$$
V^{\boldsymbol{\lambda}}(\mathbf{R}, \boldsymbol{\lambda})=\frac{1}{2} \sum_{k}^{N_{\text {sites }}} \sum_{i}^{n_{k}} \sum_{j}^{n_{k}}\left(1-\lambda_{k}\right) \frac{q_{i}^{A} q_{j}^{A}}{4 \pi \epsilon_{0} r_{i j}}+\lambda_{k} \frac{q_{i}^{B} q_{j}^{B}}{4 \pi \epsilon_{0} r_{i j}}
$$

From equation 8 the gradient with respect to $\lambda_{k}$ is:

$$
\begin{aligned}
\frac{\partial}{\partial \lambda_{k}} V_{\text {Coul }}(\mathbf{R}, \boldsymbol{\lambda})= & V_{\text {Coul }}\left(\mathbf{R}, \boldsymbol{\lambda}^{\prime}, \lambda_{k}=1\right)-V_{\text {Coul }}\left(\mathbf{R}, \boldsymbol{\lambda}^{\prime}, \lambda_{k}=0\right) \\
= & \sum_{i}^{n_{k}} \sum_{j}^{n_{\text {rest }}} \frac{q_{i}^{B} q_{j}}{4 \pi \epsilon_{0} r_{i j}}-\frac{q_{i}^{A} q_{j}}{4 \pi \epsilon_{0} r_{i j}} \\
& +\sum_{m}^{N_{\text {sites }}} \sum_{i}^{n_{k}} \sum_{j}^{n_{m}} \frac{q_{i}^{B}\left[\left(1-\lambda_{m}\right) q_{j}^{A}+\lambda_{m} q_{j}^{B}\right]}{4 \pi \epsilon_{0} r_{i j}}-\frac{q_{i}^{A}\left[\left(1-\lambda_{m}\right) q_{j}^{A}+\lambda_{m} q_{j}^{B}\right]}{4 \pi \epsilon_{0} r_{i j}} \\
& +\frac{1}{2} \sum_{i}^{n_{k}} \sum_{j}^{n_{k}} \frac{q_{i}^{B} q_{j}^{B}}{4 \pi \epsilon_{0} r_{i j}}-\frac{q_{i}^{A} q_{j}^{A}}{4 \pi \epsilon_{0} r_{i j}}
\end{aligned}
$$

Thus, the evaluation of the Coulomb contribution to the gradient for each $\lambda_{k}$-group requires two electrostatic computations, with the interpolated partial charges of the other $\lambda_{m}$ sites (i.e., $\left.q_{j}\left(\lambda_{m}\right)=\left(1-\lambda_{m}\right) q_{j}^{A}+\lambda_{m} q_{j}^{B}\right)$ :

$$
\begin{aligned}
\frac{\partial}{\partial \lambda_{k}} V_{\text {Coul }}(\mathbf{R}, \boldsymbol{\lambda})= & \sum_{i}^{n_{k}} q_{i}^{B} \times\left[\sum_{a}^{n_{\text {rest }}} \frac{q_{a}}{4 \pi \epsilon_{0} r_{i a}}\right. \\
& \left.+\sum_{m}^{N_{\text {sites }}} \sum_{j}^{n_{m}} \frac{\left(1-\lambda_{m}\right) q_{j}^{A}+\lambda_{m} q_{j}^{B}}{4 \pi \epsilon_{0} r_{i j}}+\frac{1}{2} \sum_{j}^{n_{k}} \frac{q_{j}^{B}}{4 \pi \epsilon_{0} r_{i j}}\right] \\
& -\sum_{i}^{n_{k}} q_{i}^{A} \times\left[\sum_{a}^{n_{\text {rest }}} \frac{q_{a}}{4 \pi \epsilon_{0} r_{i a}}\right. \\
& \left.+\sum_{m}^{N_{\text {sites }}} \sum_{j}^{n_{m}} \frac{\left(1-\lambda_{m}\right) q_{j}^{A}+\lambda_{m} q_{j}^{B}}{4 \pi \epsilon_{0} r_{i j}}+\frac{1}{2} \sum_{j}^{n_{k}} \frac{q_{j}^{A}}{4 \pi \epsilon_{0} r_{i j}}\right] \\
= & \sum_{i}^{n_{k}} q_{i}^{B} \Phi_{k}^{B}\left(\mathbf{R}_{i}, \boldsymbol{\lambda}^{\prime}\right)-\sum_{i}^{n_{k}} q_{i}^{A} \Phi_{k}^{A}\left(\mathbf{R}_{i}, \boldsymbol{\lambda}^{\prime}\right)
\end{aligned}
$$

Here, we introduced the electrostatic potential $\Phi_{k}^{A}\left(\mathbf{R}_{i}, \boldsymbol{\lambda}^{\prime}\right)$ of the system with partial charges 
of $\lambda$-group $k$ in the protonated state $\left(q_{i}^{A}\right)$ and interpolated charges for all other $\lambda$-groups. As before, $\boldsymbol{\lambda}^{\prime}$ is the vector with all $\lambda_{m}$ 's except $\lambda_{k}$. Likewise, electrostatic potential $\Phi_{k}^{B}\left(\mathbf{R}_{i}, \boldsymbol{\lambda}^{\prime}\right)$ is evaluated with the partial charges of $\lambda$-group $k$ in the deprotonated state $\left(q_{i}^{B}\right)$ and the same interpolated charges for all other $\lambda$-groups. Thus, $2 N_{\text {sites }}$ computations are needed to evaluate the gradients for all titratable sites. The same arguments apply to pair-wise Lennard-Jones interactions, but because the contribution of Lennard-Jones interaction to $\mathrm{p} K_{\mathrm{a}}$ shift is minor, we neglected them in this work (see Supporting Information).

\section{Linear interpolation of partial charges}

While the linear scaling of the gradients for the pair-wise potentials in equation 15 in principle is a great improvement over the formal exponential scaling in equation 8 , the requirement of performing $2 N_{\text {sites }}$ calculations per MD step still poses a computational bottleneck, in particular for larger systems. To overcome this bottleneck for electrostatic interactions, we follow the suggestion by Brooks and co-workers to interpolate charges rather than interaction functions. ${ }^{14}$ When interpolating the partial charges between the protonation states of $N_{\text {sites }}$ chemically uncoupled titratable sites, the $\lambda$-dependent Coulomb energy becomes:

$$
\begin{aligned}
& V_{\text {coul }}(\mathbf{R}, \boldsymbol{\lambda})=V^{\text {rest-rest }}(\mathbf{R})+V^{\boldsymbol{\lambda} \text {-rest }}(\mathbf{R}, \boldsymbol{\lambda})+V^{\boldsymbol{\lambda}-\boldsymbol{\lambda}}(\mathbf{R}, \boldsymbol{\lambda})+V^{\boldsymbol{\lambda}}(\mathbf{R}, \boldsymbol{\lambda}) \\
& =\frac{1}{2} \sum_{i}^{n_{\text {rest }}} \sum_{j}^{n_{\text {rest }}} \frac{q_{i} q_{j}}{4 \pi \epsilon_{0} r_{i j}} \\
& +\sum_{k}^{N_{\text {sites }}} \sum_{i}^{n_{k}} \sum_{i}^{n_{\text {rest }}} \frac{\left(\left(1-\lambda_{k}\right) q_{i}^{A}+\lambda_{k} q_{i}^{B}\right) q_{j}}{4 \pi \epsilon_{0} r_{i j}} \\
& +\sum_{k}^{N_{\text {sites }}} \sum_{m ; m \neq k}^{N_{\text {sites }}} \sum_{i}^{n_{k}} \sum_{j}^{n_{m}} \frac{\left[\left(1-\lambda_{k}\right) q_{i}^{A}+\lambda_{k} q_{i}^{B}\right]\left[\left(1-\lambda_{m}\right) q_{j}^{A}+\lambda_{m} q_{j}^{B}\right]}{4 \pi \epsilon_{0} r_{i j}} \\
& +\frac{1}{2} \sum_{k}^{N_{\text {sites }}} \sum_{i}^{n_{k}} \sum_{j}^{n_{k}} \frac{\left[\left(1-\lambda_{k}\right) q_{i}^{A}+\lambda_{k} q_{i}^{B}\right]\left[\left(1-\lambda_{k}\right) q_{j}^{A}+\lambda_{k} q_{j}^{B}\right]}{4 \pi \epsilon_{0} r_{i j}}
\end{aligned}
$$


The gradient of the potential energy with respect to $\lambda_{k}$ is

$$
\begin{aligned}
\frac{\partial V_{\text {coul }}(\mathbf{R}, \boldsymbol{\lambda})}{\partial \lambda_{k}}= & \sum_{i}^{n_{k}} \sum_{j}^{n_{\text {rest }}} \frac{\left(q_{i}^{B}-q_{i}^{A}\right) q_{j}}{4 \pi \epsilon_{0} r_{i j}} \\
& +\sum_{m=1 ; m \neq k}^{N_{\text {sites }}} \sum_{i}^{n_{k}} \sum_{j}^{n_{m}} \frac{\left(q_{i}^{B}-q_{i}^{A}\right)\left[\left(1-\lambda_{m}\right) q_{j}^{A}+\lambda_{m} q_{j}^{B}\right]}{4 \pi \epsilon_{0} r_{i j}} \\
& +\sum_{i}^{n_{k}} \sum_{j}^{n_{k}} \frac{\left(q_{i}^{B}-q_{i}^{A}\right)\left[\left(1-\lambda_{k}\right) q_{j}^{A}+\lambda_{k} q_{j}^{B}\right]}{4 \pi \epsilon_{0} r_{i j}} \\
= & \sum_{i}^{n_{k}} \sum_{j}^{n_{\text {rest }}} \frac{\left(q_{i}^{B}-q_{i}^{A}\right) q_{j}}{4 \pi \epsilon_{0} r_{i j}} \\
& +\sum_{m=1}^{N_{\text {sites }}} \sum_{i}^{n_{k}} \sum_{j}^{n_{m}} \frac{\left(q_{i}^{B}-q_{i}^{A}\right)\left[\left(1-\lambda_{m}\right) q_{j}^{A}+\lambda_{m} q_{j}^{B}\right]}{4 \pi \epsilon_{0} r_{i j}} \\
= & \sum_{i}^{n_{k}} \Phi\left(\mathbf{R}_{\mathbf{i}}, \boldsymbol{\lambda}\right) \Delta q_{i}
\end{aligned}
$$

where $\Phi\left(\mathbf{R}_{\mathbf{i}}, \boldsymbol{\lambda}\right)$ is the electrostatic potential at the position of atom $i$ due to the charge distribution of all other atoms in the system, including the atoms of all titratable sites, for which the partial charges are interpolated:

$$
\Phi\left(\mathbf{R}_{\mathbf{i}}, \boldsymbol{\lambda}\right)=\sum_{j}^{n_{\text {rest }}} \frac{q_{j}}{4 \pi \epsilon_{0} r_{i j}}+\sum_{m}^{N_{\text {sites }}} \sum_{j}^{n_{m}} \frac{\left(1-\lambda_{m}\right) q_{j}^{A}+\lambda_{m} q_{j}^{B}}{4 \pi \epsilon_{0} r_{i j}}
$$

and $\Delta q_{i}$ is the difference between the atomic charges of titratable residue $i$ in the protonated (A) and deprotonated (B) states:

$$
\Delta q_{i}=q_{i}^{B}-q_{i}^{A}
$$

In contrast to when potential energy functions are interpolated, the same electrostatic potential is used to evaluate the electrostatic forces on both the atoms and the $\lambda$-particles. Therefore, a single electrostatic calculation per time step suffices. If the electrostatic interactions are modeled with the smooth Particle Mesh Ewald method, ${ }^{36,37}$ the short-range real-space interactions and long-range reciprocal-space interactions are computed separately. For the 
pair-wise short-range interactions in real space an additional calculation for each interacting pair and a subsequent accumulation of the potential at each atom is needed. Whereas this calculation comes at no extra computational cost if the standard pair interaction kernels are used, the accumulation leads to a measurable computational overhead, as we will show later. For the mesh part of the PME calculation, a gathering of potentials from the grid is required for charges in $\lambda$-groups only, but this also comes at a negligible computational overhead. Because the extra effort required to compute the gradients on the $\lambda$ particles is rather small, a constant $\mathrm{pH}$ MD implementation based on charge interpolation is computationally not much more expensive than a normal MD simulation, which is a major improvement with respect to the previous CpHMD implementation in GROMACS. ${ }^{16}$

\section{Multisite representation of chemically coupled titratable sites}

If titratable sites are "chemically" or "topologically" coupled, the force field parameters of one site depend on the value of the $\lambda$ coordinate of the other site, and vice versa. For example, histidine can exist in three protonation states, as shown in Figure 2. In most force fields, the partial charges of all atoms in the His side chain, including the two sites, depend on the protonation state. Hence, if the $\mathrm{N}_{\delta}$ site changes protonation, also the electrostatic interactions of the $\mathrm{N}_{\epsilon}$ site are affected.

To model the chemically coupled sites in the histidine side chain, Khandogin and Brooks introduced two $\lambda$-coordinates: ${ }^{15}$ one that interpolates between the double and single protonated forms and a second coordinate switching between protonation at the $\mathrm{N}_{\delta}$ and the $\mathrm{N}_{\epsilon}$ atoms. Donnini et al. introduced separate $\lambda$-coordinates for $\mathrm{N}_{\delta}$ and $\mathrm{N}_{\epsilon} \cdot{ }^{16}$ In both solutions, the coupling between the coordinates is achieved with a two-dimensional correction potential.

Because extending the dimensionality beyond two coordinates is difficult from both the implementation and parameterization perspective, Brooks and co-workers introduced a mul-

tisite representation, ${ }^{38,39}$ where a separate $\lambda_{i, k}$-coordinate is assigned to each physical state 
$k$ of a titratable group $i$. For a residue with multiple "chemically"-coupled titratable sites each $\lambda_{i, k}$-coordinate has the same state at $\lambda_{i, k}=0$, while at $\lambda_{i, k}=1$ the group is in one of the $n_{i}$ possible protonation states (i.e., state $k$ ) of residue $i$. The state at $\lambda_{i, k}=0$ (for all coordinates $k$ of residue $i$ ) is the same for all $\lambda$-coordinates but does not correspond to a physical protonation state of the residue, and neither do states for which the sum of the $\lambda_{i, k}$-s is not equal to 1 (Figure 2). To restrict sampling to the (hyper-)plane connecting the physical states, the sum of $\lambda_{i, k}$-s is constrained $\left(\sum_{k} \lambda_{i, k}=1\right)$. Since the $\lambda$-dynamics implementation in a fork of GROMACS relies on linear $\lambda$-coordinates, rather than the auxiliary circular coordinates that would fulfill the constraints by construction, ${ }^{38}$ we apply a constraint on the sum of $\lambda$-s. To efficiently apply this constraint we use an analytical expression to solve a generalized version of charge constraint introduced by Donnini et al. ${ }^{34}$ (see Appendix). While an atom can be part of multiple $\lambda_{i, k}$ coordinates in residue $i$, each affecting its charge, we show in SI that the expression for the contribution of this atom to the total Coulomb energy is identical to that of an uncoupled site (equation 17).

In the multisite representation, each $\lambda$-coordinate is independent of the others and thus evolves on a one dimensional potential (equation 4), similar to that of "chemically" uncoupled sites. However, in contrast to the uncoupled sites, the correction potential $V^{\mathrm{MM}}$ is multidimensional as its value depends on all $\lambda_{i, k}$ coordinates representing each of the possible protonation states of residue $i$. These potentials are obtained through a least-square fit of a multi-dimensional polynomial to the ensemble-averaged gradients of the potentials with respect to $\lambda_{i, k}$ evaluated on the $\left(n_{i}-1\right)$-dimensional grid of the $n_{i}$ coupled $\lambda$-s, $i . \quad e$ : $\left\langle\partial V / \partial \lambda_{i, k}\right\rangle_{\lambda_{1} \ldots \lambda_{n_{i}}}$. The fitting procedure is explained in detail in the SI.

The multisite representation can be applied to residues with any number of titratable sites, including residues with only a single titratable site. In the latter case, two $\lambda$ coordinates, corresponding to the protonated state $\left(\lambda_{i, 1}=1, \lambda_{i, 2}=0\right)$ and deprotonated state $\left(\lambda_{i, 1}=0, \lambda_{i, 2}=1\right)$ are introduced with a constraint on their sum $\left(\lambda_{i, 1}+\lambda_{i, 2}=1\right)$. 


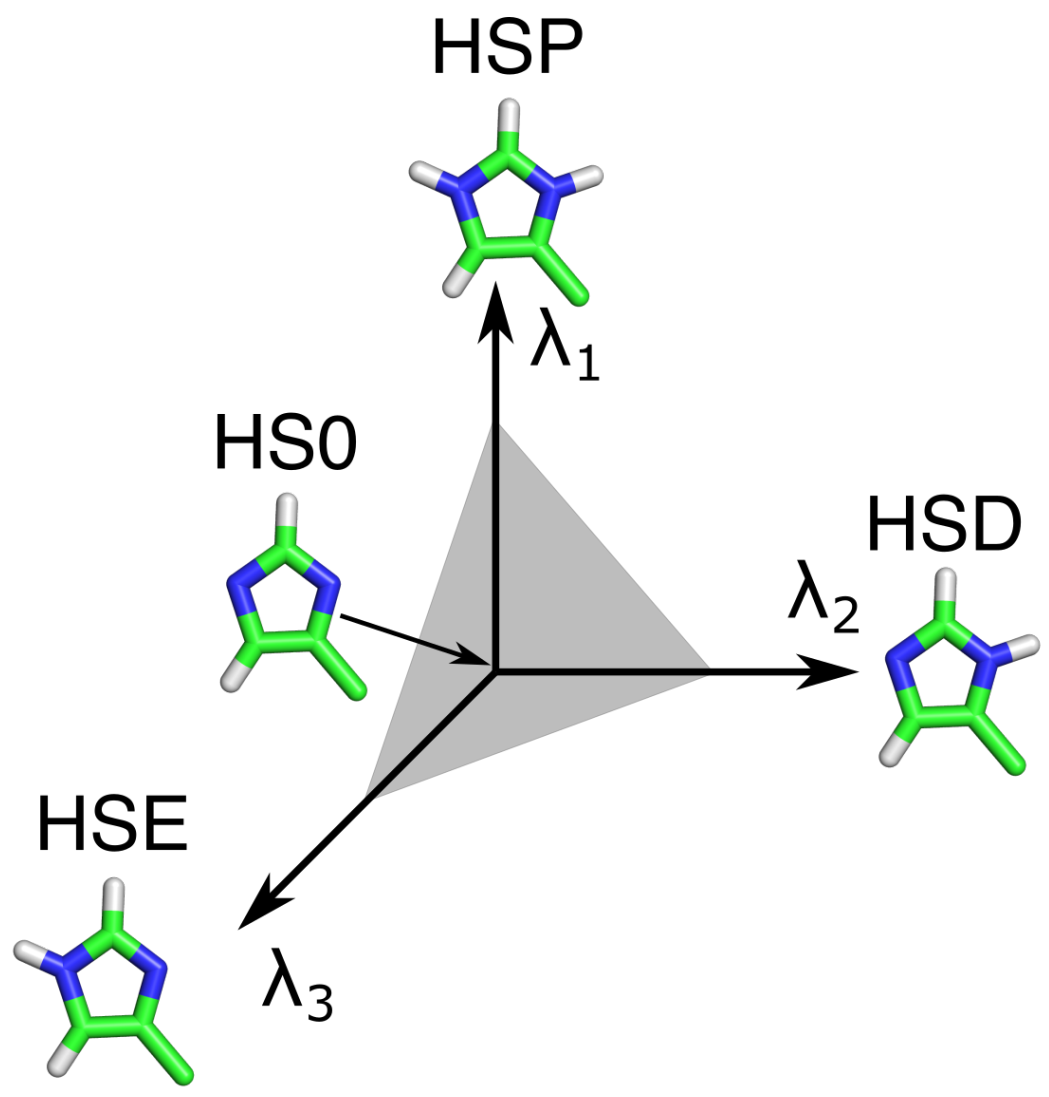

Figure 2: Multisite representation illustrated for a histidine side chain. Each possible protonation state is represented by its own $\lambda$-coordinate. HSP refers to doubly protonated histidine, HSD and HSE to histidine singly protonated at the $N_{\delta}$ or $N_{\epsilon}$ respectively. HS0 is a common, non-physical state of the residue. To restrict the sampling to the plane connecting the physical states, a constraint $\lambda_{1}+\lambda_{2}+\lambda_{3}=1$ is applied (gray plane). A biasing potential is also applied to enhance sampling at the end states, where one of the $\lambda$-coordinates is close to one, while the other coordinates are close to zero.

\section{Methods}

We have implemented the algorithms for CpHMD with charge interpolation in a fork of GROMACS software package (2021 release). ${ }^{40}$ The code and manuals are available for free at https://gitlab.com/gromacs-constantph/constantph. Here we verify the validity of our implementation for reproducing $\mathrm{p} K_{\mathrm{a}}$ values of peptides and proteins. To demonstrate that the linear interpolation of charges (equation 17) scales better with the number of titratable sites in the system than the linear interpolation of interaction functions (equation 15), we compared the scaling between our new implementation, which is based on linear charge 
interpolation on the one hand, and a previous implementation in a fork of the GROMACS 3.3 release, which is based on linear interpolation of the force field potentials on the other hand. ${ }^{16}$ To estimate the additional computational effort required for performing CpHMD with the new implementation, we also compared the performance of a CpHMD simulation to that of a normal MD simulations on both CPUs and GPUs.

\section{Simulated systems}

To test the implementation, we performed constant $\mathrm{pH}$ MD simulations of the following systems:

1. Glutamic acid (Glu)

2. Aspartic acid (Asp)

3. Histidine (His)

4. Cardiotoxin V (PDB ID: $1 \mathrm{CVO}^{41}$ )

5. Hen egg white lyzozyme (HEWL, PDB ID: 2LZT ${ }^{42}$ )

6. The GLIC pentameric Ligand-Gated Ion Channel (PDB ID: 4HFI ${ }^{43}$ )

7. Turkey ovomucoid inhibitor (PDB ID: $2 \mathrm{GKR}^{44}$ )

In systems 1-6 the interactions were modelled with the CHARMM36 ${ }^{45}$ all-atom (AA) force field, with some modifications in the torsion parameters to accelerate the convergence. These modifications are presented and validated in an accompanying paper. ${ }^{46}$ Systems 1-5 were also simulated with the Martini 2.0 coarse grained (CG) force field. ${ }^{33}$ The martinize.py script was used to automatically generate the CG representation of these systems. ${ }^{47}$ System 7 was simulated to compare the efficiency of interpolating charges and potentials. The interactions in this system were modeled with the OPLS force field, ${ }^{48}$ because the GROMACS 
3.3 release, on which the linear interpolation of potentials implementation was based, does not support the CMAP correction that is needed for the CHARMM36 force field. ${ }^{49}$

The amino acids Glu, Asp and His were modelled as tripeptides Ala-X-Ala with acetylated N-terminus (ACE) and N-methylamidated C-terminus $\left(\mathrm{CT}_{3}\right)$. The proteins were simulated with charged termini. The tripeptides were placed in a periodic rectangular box of dimensions $5 \times 5 \times 5 \mathrm{~nm}^{3}$ with approximately 4000 CHARMM TIP3P $\mathrm{P}^{50,51}$ water molecules for the AA simulations and 950 polarizable water particles for the CG simulations. ${ }^{52}$ The water soluble protein cardiotoxin $\mathrm{V}$ was placed in a periodic rectangular box of $7.9 \times 7.9 \times 7.9 \mathrm{~nm}^{3}$ and filled with 16500 CHARMM TIP3P water molecules for the AA simulations. For the CG simulations, the protein was placed inside a periodic rectangular box of $5.7 \times 5.7 \times 5.7 \mathrm{~nm}^{3}$ and filled with 1800 polarizable water particles. The larger water soluble protein HEWL was placed in a periodic rectangular box of $8.9 \times 8.9 \times 8.9 \mathrm{~nm}^{3}$ and filled with 23000 CHARMM TIP3P water molecules for the AA simulations and 5400 polarizable water particles for the CG simulations. $\mathrm{Na}^{+}$and $\mathrm{Cl}^{-}$ions were added to all systems at $0.15 \mathrm{M}$ concentration to neutralize the protein systems. The turkey ovomucoid inhibitor protein was placed in a box of $4.9 \times 4.9 \times 4.9 \mathrm{~nm}^{3}$ with $3086 \mathrm{SPC}^{53}$ water molecules. The GLIC protein was embedded into a bilayer membrane containing 498 phosphatidylcholine (POPC) lipids, placed in a box of $14.0 \times 14.0 \times 15.9 \mathrm{~nm}^{3}$, and filled with 66494 CHARMM TIP3P waters, $58 \mathrm{Na}^{+}, 123$ $\mathrm{Cl}^{-}$ions. The system contained 292135 atoms in total. The simulation of this system was performed with the GROMACS 2021.4 release as reference. The GLIC bencnhmarks were run with default settings on an Intel i9-7920X 12-core CPU and an Nvidia RTX 2080 Ti GPU. All input configurations are provided as Supporting Information.

In the AA simulations, Coulomb interactions were modeled with the smooth PME method with a real-space cut-off of $1.2 \mathrm{~nm}$ and a grid spacing of $0.14 \mathrm{~nm},{ }^{36,37}$ while Lennard-Jones interactions were smoothly switched to zero in range from 1.0 to $1.2 \mathrm{~nm}$. In the CG simulations, Coulomb interactons were modeled by a Reaction Field potential with a $1.1 \mathrm{~nm}$ cut-off, $\epsilon_{\mathrm{r}}=2.5$, and $\epsilon_{\mathrm{RF}}=\infty$, while Lennard-Jones interactions were truncated at $1.1 \mathrm{~nm} .{ }^{54}$ 
To keep the temperature constant at $300 \mathrm{~K}$, we used the v-rescale thermostat ${ }^{55}$ with time constants of 0.5 and $1.0 \mathrm{ps}^{-1}$ for AA and CG simulations, respectively. The pressure was kept constant at 1 bar with the Parrinello-Rahman barostat ${ }^{56}$ with relaxation times of 2.0 and 12.0 ps for AA and CG simulations, respectively. A leapfrog integrator was used with an integration steps of 2 an 20 fs for AA and CG simulations, respectively. In the AA simulations, the LINCS algorithm ${ }^{57}$ was used to constrain bond lengths of the solutes, while the SETTLE $^{58}$ algorithm was used to constrain internal degrees of freedom of the water molecules. Prior to the constant $\mathrm{pH}$ MD simulations, the potential energy of each system was minimized using the steepest descent method, followed by 1 ns of equilibration.

\section{Constant pH MD simulation setups}

In the atomistic simulations the multisite representation was used to model the protonation states of titratable residues. Two $\lambda$-coordinates were introduced to model the two forms of the carboxylic acid side chain in Asp and Glu, while three coordinates were used to describe the three protonation states of the imidazole sidechain in His. In the CG simulations, the single-site representation was used, in which the A and B states represent the protonated and deprotonated states of the titratable beads. Because, in contrast to AA force fields, there is no distinction between the two neutral forms of the His sidechain in the Martini force

field, the single-site description for HIS suffices in the CG simulations. In both atomistic and coarse-grained simulations, the transformations between the different protonation states were achieved by changing the charges of the ionizable groups. The Lennard-Jones and bonded terms (bonds, angles, and torsions) were kept in the protonated and deprotonated state in AA and CG simulations, respectively (see Supplementary Information, Fig. S3). We show in SI that the contribution of these terms is sufficiently small to be neglected without significant error. Note, that these terms can be made $\lambda$-dependent as well, but this is beyond the scope of the current work since the efforts to implement this are high.

The mass of the $\lambda$-particles was set to 5 atomic units, and their temperature was main- 
tained at $300 \mathrm{~K}$ by using a separate v-rescale thermostat for the $\lambda$ coordinates with a time constant of $2.0 \mathrm{ps}^{-1}$. For all $\lambda$-coordinates the biasing potential $V_{i}^{\text {bias }}\left(\lambda_{i}\right)$ was defined by equation S1 in the SI. The barrier height of the double well potential was set to 5.0 and $7.5 \mathrm{~kJ} / \mathrm{mol}$ for AA and CG simulations, respectively. The parameters for the double well potential and the $\mathrm{pH}$ dependent potential (Equation 4) are provided in Table S1 in the SI.

For the tripeptides, we calculated five independent CpHMD trajectories of 20 ns each at $13 \mathrm{pH}$ values, ranging from 1.0 to 7.0 for the peptides with Glu and Asp, and from 4.0 to 10.0 for the peptides with His. For the cardiotoxin V protein (three Asp and one His titratable residues), we performed five independent CpHMD simulations of 50 ns at $15 \mathrm{pH}$ values between 1.0 to 8.0. For the HEWL protein (seven Asp, two Glu and one His titratable residues), we performed five independent CpHMD simulations of 75 ns at $21 \mathrm{pH}$ values between -1.0 to 9.0 . The values of the $\lambda$-coordinates were written to file with a frequency of $1 \mathrm{ps}^{-1}$.

\section{Reference states and force field correction potentials}

The constant $\mathrm{pH}$ simulations of the aforementioned systems require reference states for Asp, Glu and His, in which the proton affinity $\left(\mathrm{p} K_{\mathrm{a}}\right)$ is known from experiment. The measured and calculated (force field) deprotonation free energies of these reference states were used to include the effect of the $\mathrm{pH}$ bath, as well as the effects of the breaking and forming of chemical bonds in the simulation, i.e., $V^{\mathrm{MM}}$ in Equation 2. The measured reference $\mathrm{p} K_{\mathrm{a}}$ values used in this work are included in Table 1 . Note that the experimental values were obtained for pentapeptides, while tripeptides were used for computing $V^{\mathrm{MM}}$. This however did not affect the results, as shown in Figure S2 of SI.

Thermodynamic integration was used to compute the reference free energies as follows: The partial charges in tripeptide systems representing the reference states of Glu, Asp, His were linearly interpolated between $\lambda=-0.1$ and $\lambda=1.1$ with a step of 0.05 under the constraint $\lambda_{1}+\lambda_{2}=1$ for Glu and Asp, while for His, the constraint was $\lambda_{1}+\lambda_{2}+\lambda_{3}=1$. 
For each set of $\lambda$ values, called a grid point, a $10 \mathrm{~ns}$ MD simulation was performed. $\partial V / \partial \lambda_{i}$ were saved every ps, which is approximately equal to the auto-correlation times for the $\lambda$-coordinates. The total charge of the system was kept neutral by simultaneously changing the charge of a single buffer particle, discussed below. The $\partial V / \partial \lambda_{i}$ values were averaged over the last $9 \mathrm{~ns}$ of the trajectories. To obtain an analytical expression for $V^{\mathrm{MM}}$ a fifth order polynomial was fitted to these averages for Asp and Glu, while an eighth order polynomial was fitted for His, taking into account possible linear dependencies of the coefficients (see Supplementary Information). Fitting errors were below $0.5 \mathrm{~kJ} / \mathrm{mol}$ for Asp and Glu and below $1 \mathrm{~kJ} / \mathrm{mol}$ for His, which are of similar magnitude as the statistical accuracy of the derivatives.

\section{Buffer particles}

Dynamically changing partial charges can affect the total charge of the simulation unit cell, which can lead to artifacts, as documented for instance in Hub et al. for Ewald-based methods. ${ }^{59}$ To avoid such artifacts, it is essential to keep the total charge of the unit cell constant. Two approaches have been proposed: (i) direct coupling between each titratable residue and a water,${ }^{20}$ or ion, ${ }^{18}$ and (ii) titratable buffers that collectively compensate changes in charge of all titratable residues. ${ }^{34}$

Here, we follow the latter approach, but with several improvements for all-atom simulations. Firstly, to avoid restraints, which were needed to minimize interactions between the buffers and the titrable sites in previous work, ${ }^{34}$ we introduced buffer particles with both small LJ radius and small partial charges of maximal $|0.5| e$, such that they do not disturb the hydrogen bond network, nor interact too strongly with the titratable sites or other buffers. Secondly, to also prevent strong interactions with hydrophobic regions in the system, the C6 dispersion parameter with anything other than water was set to zero, including the other buffers. The latter also avoids clustering of buffers during the simulation. Further details on buffer parameterization are provided in the accompanying paper. ${ }^{46}$ Thus, the buffer particles 
have an $\sigma$ of $0.25 \mathrm{~nm}$ and an $\epsilon$ of $4 \mathrm{kJmol}^{-1}$. In coarse-grained simulations, standard $\mathrm{Na}^{+}$ ions were used as buffer particles.

As in Donnini et al. ${ }^{34}$ the buffers were collectively coupled to the titratable sites in the system via a charge constraint. The charges of all buffers were thus simultaneously interpolated between $-0.5 e$ and $0.5 e$, keeping the simulation box neutral. For all peptide simulations, 10 such buffers were introduced into the system, while 20 buffers were added to the simulation boxes with the proteins (systems 4-5), in both AA and CG models. 185 buffer particles were used in GLIC simulations.

\section{Analysis of the constant $\mathrm{pH}$ trajectories}

To estimate the $\mathrm{p} K_{\mathrm{a}}$ values of titratable groups from multiple simulations at various $\mathrm{pH}$ values, we computed the average fraction of deprotonated frames $\left(S^{\text {deprot}}\right)$ over all replicas. For a group with a single titratable site, this average was obtained as

$$
S^{\text {deprot }}(\mathrm{pH})=\frac{N^{\text {deprot }}}{N^{\text {prot }}+N^{\text {deprot }}}
$$

where $N^{\text {prot }}$ and $N^{\text {deprot }}$ are the total number of frames in which the site is protonated and deprotonated, respectively. For titratable sites modeled in the single-site representation, we considered it protonated if $\lambda$ is below 0.2 , and deprotonated if $\lambda$ is above 0.8. For sites that are described with the multisite description, we considered a state protonated if the $\lambda$ associated with the protonated form of the residue is above 0.8 , and deprotonated if the $\lambda$ associated with the deprotonated form of the residue is above 0.8 .

To estimate the macroscopic $\mathrm{p} K_{\mathrm{a}}$ values of histidine, which contains two titratable sites $\mathrm{N}_{\epsilon}$ and $\mathrm{N}_{\delta}$, we calculated for each $\mathrm{pH}$ value the average fraction of frames in which the residue is deprotonated at either of the two sites:

$$
S_{\text {macro }}^{\text {deprot }}(\mathrm{pH})=1-\frac{N^{\lambda_{p}}}{N^{\lambda_{p}}+N^{\lambda_{\epsilon}}+N^{\lambda_{\delta}}}
$$


where $N^{\lambda_{p}}, N^{\lambda_{\epsilon}}$ and $N^{\lambda_{\delta}}$ are the numbers of frames in which $\lambda_{p}>0.8, \lambda_{\epsilon}>0.8$ and $\lambda_{\delta}>0.8$ (Figure 2). To estimate the microscopic $\mathrm{p} K_{\mathrm{a}}$ values for the two sites of His, we calculated for each site the average fraction of frames in which that site was deprotonated:

$$
S_{\operatorname{micro}(\delta / \epsilon)}^{\mathrm{deprot}}(\mathrm{pH})=\frac{N^{\lambda_{\delta} / \lambda_{\epsilon}}}{N^{\lambda_{p}}+N^{\lambda_{\epsilon} / \lambda_{\delta}}}
$$

Errors were estimated from the standard error of the mean for the different replicas.

The averaged fractions at each pH value were fitted to the Henderson-Hasselbalch equation:

$$
S^{\text {deprot }}=\frac{1}{10^{\left(\mathrm{p} K_{\mathrm{a}}-\mathrm{pH}\right)}+1}
$$

which yielded the $\mathrm{p} K_{\mathrm{a}}$ values as fitting parameters. The error in the $\mathrm{p} K_{\mathrm{a}}$ was estimated from $95 \%$ confidence interval for the non-linear least squares fit to the average $\left(S^{\text {deprot}}\right)$ values.

\section{Results and Discussion}

Here we discuss the results obtained with our new implementation of constant $\mathrm{pH}$ into the fork of GROMACS 2021 release. ${ }^{40}$

\section{Titration of single amino acids}

In Figure 3 we show the titration curves for AlaAspAla, AlaGluAla and AlaHisAla tripeptides, obtained from simulations with the modified all-atom CHARMM36 ${ }^{46}$ and coarse-

grained Martini 2.0 force fields. ${ }^{33}$ Fitting the deprotonated fractions as a function of $\mathrm{pH}$ value to the Henderson-Hasselbalch equation (dashed lines in Figure 3) yields $\mathrm{p} K_{\mathrm{a}}$ values for the tripeptides that are within $0.1 \mathrm{p} K_{\mathrm{a}}$ units from the reference values. Comparing the titration curves obtained with the Martini 2.0 force field in our implementation to those com-

puted with the constant $\mathrm{pH}$ approach developed explicitly for this coarse-grained model, ${ }^{26}$ our results suggest a much better agreement with experiment than the latter. We attribute 
this difference to the more sophisticated explicit treatment of proton-like particles in the Martini constant $\mathrm{pH}$ approach. The rather good agreement between the titration curves obtained for both force fields on the one hand and experiment on the other hand suggests that our implementation has little to no dependency on the force field, in line with the GROMACS philosophy of supporting a wide range of popular force fields.
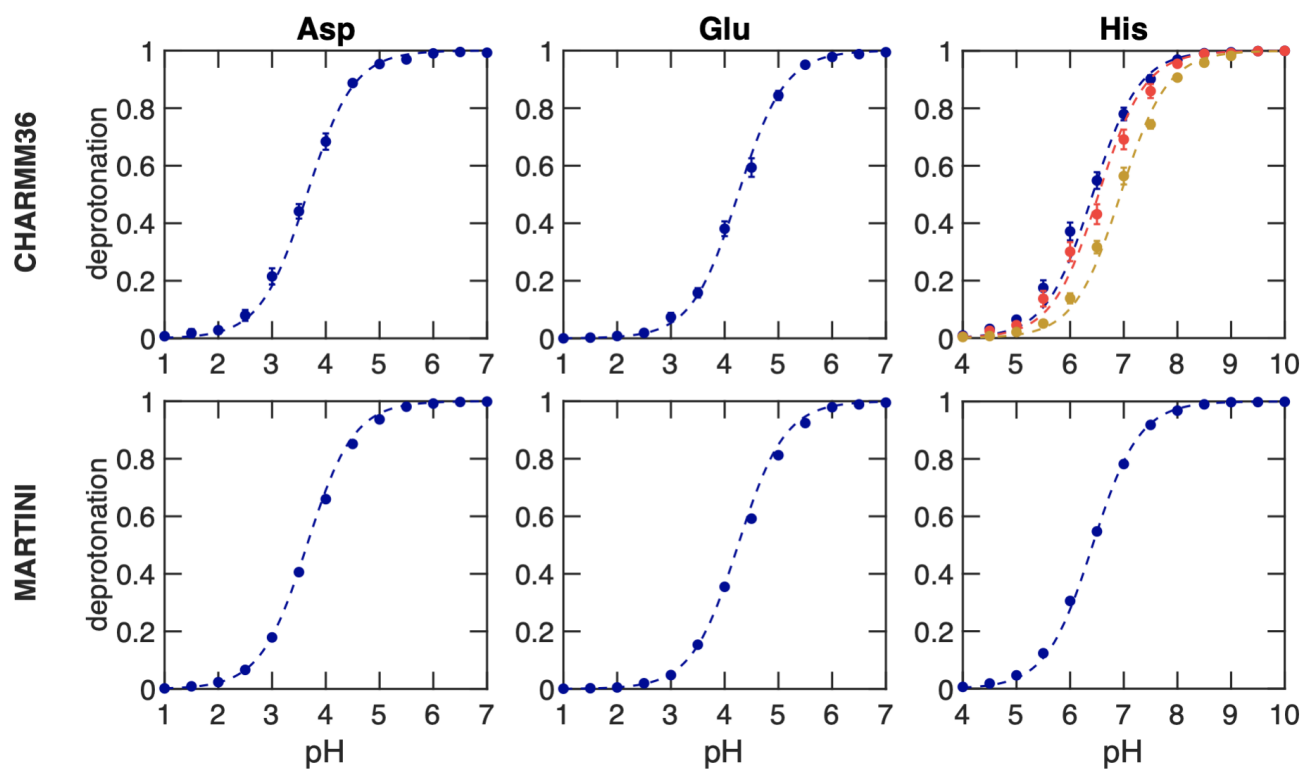

Figure 3: Titrations of tripeptide amino acids (Glu, Asp, His) in water. The top and bottom rows show titrations performed with the modified AA CHARMM $36^{46}$ and CG Martini ${ }^{33}$ force fields, respectively. In all simulations neutrality was maintained by including ten buffer particles in combination with the charge constraint. Dots show the fraction of frames in which the residue is deprotonated, and the dashed lines represent the fits to the HendersonHasselbalch equation. For His, blue color represents the macroscopic $\mathrm{p} K_{\mathrm{a}}$, while yellow and red represent the microscopic $\mathrm{p} K_{\mathrm{a}}$ values for $\mathrm{HSD}$ (proton on $\mathrm{N}_{\delta}$ ) and HSE (proton on $\mathrm{N}_{\epsilon}$ ), respectively. In the Martini 2.0 model, HSD and HSE are indistinguishable and hence only the macroscopic titration curve is shown. From the fits the $\mathrm{p} K_{\mathrm{a}}$ values were estimated and listed in Table 1.

\section{Titration of proteins}

The titration curves of cardiotoxin $\mathrm{V}$ and HEWL proteins are shown in Figures 4 and 5, respectively. The $\mathrm{p} K_{\mathrm{a}}$ values obtained from fitting the Henderson-Hasselbalch equation to the degree of deprotonation in the all-atom simulations of both proteins with the CHARMM36 
Table 1: $\mathrm{p} K_{\mathrm{a}}$ values obtained from titration simulations. The reference $\mathrm{p} K_{\mathrm{a}}$ values for tripeptides are given in the last column that contains the experimental $\mathrm{p} K_{\mathrm{a}}$ values. The values for Asp and Glu are taken from, ${ }^{60}$ while the microscopic and macroscopic $\mathrm{p} K_{\mathrm{a}}$ values for His are taken from. ${ }^{61}$ Experimentally obtained $\mathrm{p} K_{\mathrm{a}}$ values for cardiotoxin $\mathrm{V}$ are from ${ }^{62,63}$ and for HEWL from. ${ }^{64}$

\begin{tabular}{|c|c|c|c|}
\hline & \multicolumn{3}{|c|}{$\mathrm{p} K_{\mathrm{a}}$ values } \\
\hline \multirow[t]{2}{*}{ Amino acid } & CHARMM36 & MARTINI & Exp. \\
\hline & \multicolumn{3}{|c|}{ Tripeptide simulations ${ }^{60,61}$} \\
\hline Asp & $3.61 \pm 0.03$ & $3.69 \pm 0.02$ & 3.65 \\
\hline Glu & $4.26 \pm 0.04$ & $4.30 \pm 0.03$ & 4.25 \\
\hline His macroscopic & $6.34 \pm 0.08$ & $6.40 \pm 0.03$ & 6.42 \\
\hline His HSD & $6.56 \pm 0.06$ & & 6.53 \\
\hline \multirow[t]{2}{*}{ His HSE } & $6.90 \pm 0.05$ & & 6.94 \\
\hline & \multicolumn{3}{|c|}{ Simulation of cardiotoxin $\mathrm{V}^{62,63}$} \\
\hline His-4 & $5.14 \pm 0.16$ & $4.54 \pm 0.09$ & 5.5 \\
\hline Glu-17 & $4.08 \pm 0.08$ & $4.36 \pm 0.04$ & 4 \\
\hline Asp-42 & $4.02 \pm 0.10$ & $4.30 \pm 0.05$ & 3.2 \\
\hline \multirow[t]{2}{*}{ Asp-59 } & $2.41 \pm 0.07$ & $1.45 \pm 0.03$ & $<2$ \\
\hline & \multicolumn{3}{|c|}{ Simulation of HEWL ${ }^{64}$} \\
\hline Glu-7 & $2.82 \pm 0.07$ & $4.86 \pm 0.05$ & 2.6 \\
\hline His-15 & $4.84 \pm 0.05$ & $5.42 \pm 0.05$ & 5.5 \\
\hline Asp-18 & $3.35 \pm 0.05$ & $3.31 \pm 0.03$ & 2.8 \\
\hline Glu-35 & $7.64 \pm 0.13$ & $6.36 \pm 0.05$ & 6.1 \\
\hline Asp-48 & $0.99 \pm 0.07$ & $3.36 \pm 0.05$ & 1.4 \\
\hline Asp-52 & $5.69 \pm 0.12$ & $7.18 \pm 0.10$ & 3.6 \\
\hline Asp-66 & $1.70 \pm 0.10$ & $5.22 \pm 0.05$ & 1.2 \\
\hline Asp-87 & $1.73 \pm 0.03$ & $3.47 \pm 0.05$ & 2.2 \\
\hline Asp-101 & $5.43 \pm 0.11$ & $4.20 \pm 0.06$ & 4.5 \\
\hline Asp-119 & $2.77 \pm 0.05$ & $3.80 \pm 0.05$ & 3.5 \\
\hline
\end{tabular}

force field, listed in Table 1, are in good agreement with previous constant pH MD simulations, ${ }^{23,65}$ and in reasonable agreement with experimental estimates from NMR spectroscopy. ${ }^{62-64}$ In particular, the trends in the $\mathrm{p} K_{\mathrm{a}}$ shifts are well reproduced, including the downshift of Asp-59 in cardiotoxin V, and, with the exception of Glu-35 and Asp-52 in HEWL, the deviations are below $1 \mathrm{p} K_{\mathrm{a}}$ unit. We note that also in previous constant $\mathrm{pH}$ simulations with the CHARMM force field, ${ }^{15,23}$ similar deviations were found for these two residues (see Figure S5). This suggests that the origin of the discrepancy might lie beyond the implementation, and could be due to either a lack of sampling, or systematic shortcom- 
ings in the force field. The $\mathrm{p} K_{\mathrm{a}}$ values estimated from the Martini 2.0 force field simulations of these proteins do not agree as well to experiment as those derived from the all-atom simulations. We speculate that the larger deviation of the $\mathrm{p} K_{\mathrm{a}}$ 's in the coarse-grained constant $\mathrm{pH}$ simulations could be due to the lower accuracy of the electrostatic interactions. Although we still consider the results obtained with the Martini simulations reasonable, in particular for the peptides, the discrepancies for the titratable residues in proteins suggest that additional parameterization efforts may be required to systematically improve the force field for constant $\mathrm{pH}$ MD simulations based on $\lambda$-dynamics. Such improvements would be particularly worthwhile considering coarse grained simulations pave the way to perform MD simulations of complete organelles, ${ }^{66}$ in which many processes have a strong $\mathrm{pH}$ dependence.

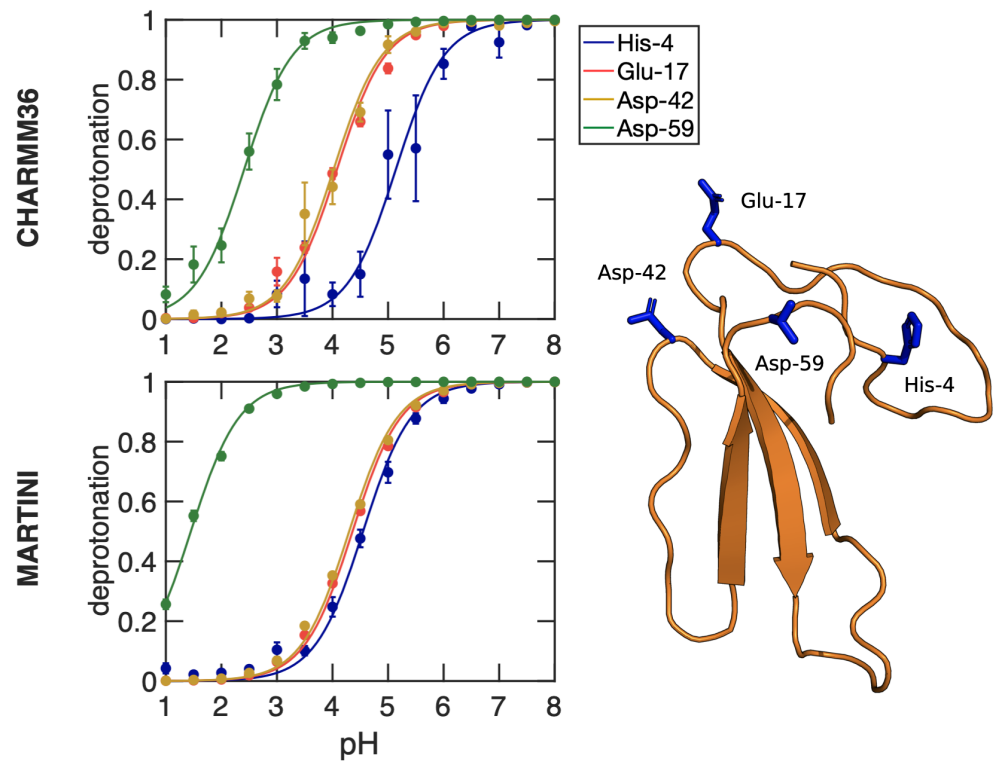

Figure 4: Titration curves of the cardiotoxin $\mathrm{V}$ protein obtained from constant $\mathrm{pH} \mathrm{MD}$ simulations with the CHARMM36 (top) and Martini 2.0 force fields (bottom). For each of the four titratable residues in this protein the dots show the fraction of frames in which the residue is deprotonated. The lines show the best fits to the Henderson-Hasselbalch equation. The $\mathrm{p} K_{\mathrm{a}}$ values for each titratable residue were estimated from these fits, and listed in Table 1. The right panel shows the protein structure with the four titratable residues highlighted in stick representation. 

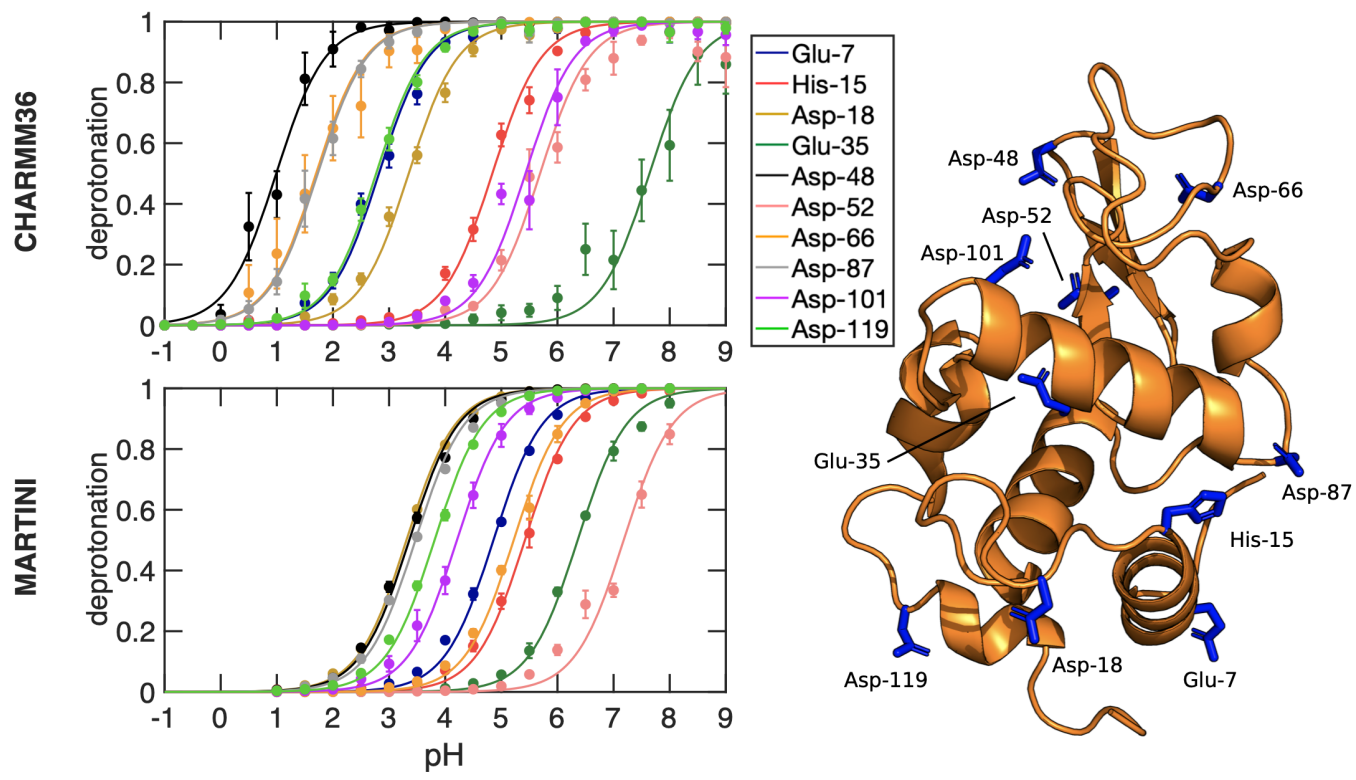

Figure 5: Titration curves of the HEWL protein obtained from constant $\mathrm{pH}$ MD simualtions with the CHARMM36 (top) and Martini 2.0 force fields (bottom). For each of the ten titratable residues, the dots show the fraction of frames in which that residue is deprotonated. The lines show the best fit to the Hendersen-Hasselbach equation. The $\mathrm{p} K_{\mathrm{a}}$ values for each titratable residue were estimated from these fits, and listed in Table 1. The right panel shows the protein structure with the ten titratable residues highlighted in stick representation.

\section{Efficiency of the implementation}

To demonstrate that linear interpolation of charges is computationally more efficient than linear interpolation of the potential energy functions for systems with many titratable sites, we investigated how the computational cost of a simulation scales with the number of titratabe sites in the system for both approaches. Because we have implemented interpolation of the charges rather than potentials into the fork of GROMACS 2021 release, whereas the potential interpolation was implemented in a fork of GROMACS 3.3 release, we compare the relative performances of both codes for an increasing number of titratable sites in the system. We define the relative performance as the ratio between the average wall-clock time per integration step for a simulation with constant $\mathrm{pH}$ on the one hand and the average wall-clock time per integration step for a normal simulation without constant $\mathrm{pH}$ on the other hand. 
Figure $6 \mathrm{~A}$ shows that relative performance of constant $\mathrm{pH}$ simulations with charge interpolation does not decrease when the number of titratable sites included in the simulation increases. Most of the 30-40\% drop in performance compared to a normal MD simulation with the same version of GROMACS is caused by the additional calculations and reductions in the non-bonded pair-interaction kernels that are required to obtain the real-space part of the electrostatic potential $\left(\Phi\left(\mathbf{R}_{\mathbf{i}}, \boldsymbol{\lambda}\right)\right.$ in Equation 17$)$.

In contrast, the relative performance of constant $\mathrm{pH}$ simulations based on the linear interpolation of potential energy functions, decreases with the number of titratable sites in the system. This comparison thus demonstrates that by replacing linear interpolation of potentials by linear interpolation of partial charges, we have overcome the major bottleneck in the earlier constant $\mathrm{pH}$ implementation in the fork of GROMACS 3.3 release, and paved the way towards simulations of large biomolecular systems at constant $\mathrm{pH}$. An example of such a large system is the proton-gated ion channel GLIC, a membrane protein with 185 titratable residues. Figure $6 \mathrm{~B}$ shows the performance of the new implementation for this large system when running the simulation on CPU and on a combination of CPU and GPU. While the computational overhead is somewhat larger when using a GPU in addition to a CPU, the overall performance still improves significantly when adding a GPU.

\section{Conclusions}

We have presented and validated a new implementation of $\lambda$-dynamics based constant $\mathrm{pH}$ molecular dynamics in the GROMACS software. Our implementation combines several developments in this field into a single MD program, including the multisite representation of titratable groups, ${ }^{38}$ charge interpolation, ${ }^{14}$ Particle Mesh Ewald electrostatics, ${ }^{23}$ and charge constraints. ${ }^{34}$ Test calculations on amino acids and proteins suggest that the new implementation is efficient, accurate and agnostic to force fields. Combined with user-friendly parameterization protocols, presented in the accompanying paper, ${ }^{46}$ we expect that this im- 

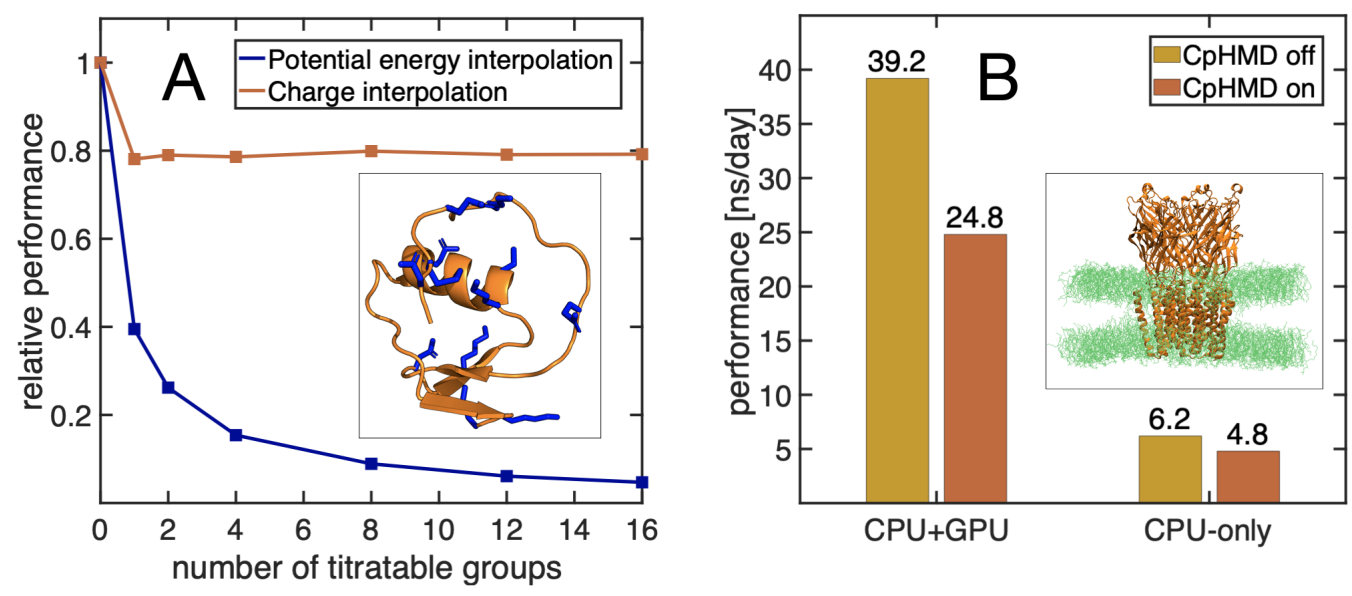

Figure 6: (A) Relative performance of interpolating potentials in a previous implementation of CpHMD into a fork of GROMACS 3.3 release (blue) and of charge interpolation in our new implementation (red) as a function of the number of titratable sites. The simulatioms were performed for the turkey ovomucoid inhibitor protein (PDB ID: $2 \mathrm{GKR}^{44}$ ), shown in the inset, where the titratable sites are highlighted in stick representation. (B) Comparison of the performance between CPU-only and CPU + GPU implementations for the ligand-gated ion channel GLIC (PDB ID: $4 \mathrm{HFI}^{43}$ ) with 185 titratable sites. In total, the GLIC system contained 292135 atoms.

plementation will pave the way towards routinely including the effect of $\mathrm{pH}$ in biomolecular MD simulations.

\section{Appendix A: Constraint algorithm}

We use constraints to restrict sampling to the correct protonation states in the multisite representation as well as to maintain a neutral charge of the simulation box. Both multisite and charge constraints keep the linear combination of a subset of $\lambda$ coordinates constant, and are applied simultaneously. Thus, there are $N_{c}$ constraint equations

$$
\sigma^{k}(\boldsymbol{\lambda})=\sum_{i=1}^{N_{\text {sites }}} \alpha_{i}^{k} \lambda_{i}=C^{k}
$$

for $k \leq N_{c}$. Here, $\boldsymbol{\lambda}$ is the vector of all $\lambda_{i}$-coordinates and $C^{k}$ is the value of constraint $k$, which can be zero. If $\sigma^{k}(\boldsymbol{\lambda})$ is a multisite constraint, $\alpha_{i}^{k}=1$ for $\lambda_{i}$-coordinates that represent 
one of the protonation states of a residue, while $\alpha_{i}^{k}=0$ for all other $\lambda_{i}$-coordinates. If $\sigma^{k}(\boldsymbol{\lambda})$ is a constraint for keeping the overall charge constant, $\alpha_{i}^{k}=\sum_{j}^{N_{\text {atoms }}^{i}} q_{j, i}^{B}-q_{j, i}^{A}$, with $N_{\text {atoms }}^{i}$ the number of atoms whose charges change as a function of $\lambda_{i}$.

During a leap-frog integration step all $\lambda_{i}$-coordinates are first propagated without contraints to their unconstrained new values $\lambda_{i}^{u}$, which do not fullfill the constraints in Equation 22. To obtain the constrained $\lambda_{i}^{c}$ values, we first connect the constrained and unconstrained $\lambda_{i}$ values using the definition of $\sigma^{k}(\boldsymbol{\lambda})$ :

$$
\sigma^{k}\left(\boldsymbol{\lambda}^{c}\right)=\sigma^{k}\left(\boldsymbol{\lambda}^{u}\right)+\sum_{i=1}^{N_{\text {sites }}} \alpha_{i}^{k}\left[\lambda_{i}^{c}-\lambda_{i}^{u}\right]
$$

Because the unconstrained and constrained $\lambda_{i}$-coordinates are also connected by the constraint forces $G_{i}^{k}=-\zeta^{k} \frac{\partial \sigma^{k}}{\partial \lambda_{i}}$, we have in addition that

$$
\lambda_{i}^{c}=\lambda_{i}^{u}+\sum_{k=1}^{N_{c}} G_{i}^{k} \frac{\Delta t^{2}}{m_{i}}=\lambda_{i}^{u}-\sum_{k=1}^{N_{c}} \zeta^{k} \frac{\partial \sigma^{k}}{\partial \lambda_{i}} \frac{\Delta t^{2}}{m_{i}}=\lambda_{i}^{u}-\sum_{k=1}^{N_{c}} \zeta^{k} \alpha_{i}^{k} \frac{\Delta t^{2}}{m_{i}}
$$

where $\zeta^{k}$ is the Lagrange multiplier for constraint $k, m_{i}$ the fictitious mass of $\lambda_{i}$ and $\Delta t$ the integration time step. Substituting Equation 24 in 23 yields

$$
\sigma^{k}\left(\boldsymbol{\lambda}^{c}\right)=\sigma^{k}\left(\boldsymbol{\lambda}^{u}\right)-\sum_{i=1}^{N_{\text {sites }}} \alpha_{i}^{k} \sum_{l=1}^{N_{c}} \zeta^{l} \alpha_{i}^{l} \frac{\Delta t^{2}}{m_{i}}
$$

which after rearranging can be expressed as

$$
\Delta \sigma^{k}=\sigma^{k}\left(\boldsymbol{\lambda}^{u}\right)-\sigma^{k}\left(\boldsymbol{\lambda}^{c}\right)=\sum_{l=1}^{N_{c}} \zeta^{l} \sum_{i=1}^{N_{\text {sites }}} \alpha_{i}^{k} \alpha_{i}^{l} \frac{\Delta t^{2}}{m_{i}}
$$

The last expression can be rewritten in matrix form

$$
\Delta \boldsymbol{\sigma}=\left(\Delta \sigma^{1}, \ldots, \Delta \sigma^{N_{c}}\right)^{T}=\mathbf{A} \boldsymbol{\zeta}
$$


where $\boldsymbol{\zeta}=\left(\zeta^{1}, \ldots, \zeta^{N_{c}}\right)^{T}$ and

$$
\mathbf{A}=\left(\sum_{i=1}^{N_{\mathrm{sites}}} \alpha_{i}^{k} \alpha_{i}^{l} \frac{\Delta t^{2}}{m_{i}}\right)
$$

Because the $\alpha_{i}^{k}$ coefficients remain the same, matrix $\mathbf{A}$ is computed once at the start of the simulation. At each step, the elements $\Delta \sigma^{k}$ are evaluated as the difference between the $\sigma^{k}\left(\boldsymbol{\lambda}^{u}\right)$ and $\sigma^{k}\left(\boldsymbol{\lambda}^{c}\right)=C^{k}$ :

$$
\Delta \sigma^{k}=\sigma^{k}\left(\boldsymbol{\lambda}^{u}\right)-C^{k}
$$

The Lagrange multipliers $\zeta^{k}$ are then obtained from Equation 27 and used to correct the unconstrained $\lambda_{i}$ values (Equation 24).

\section{Acknowledgement}

This research was supported by the Swedish Research Council (grant no. 2019-04477) and Academy of Finland (grants 311031 and 332743). The simulations were performed on resources provided by the CSC - IT Center for Science, Finland and the Swedish National Infrastructure for Computing (SNIC 2021/1-38). We also thank Erik Lindahl, Helmut Grubmüller, Dmitry Morozov, Serena Donnini and Plamen Dobrev for their support during the project.

\section{Supporting Information Available}

The Supporting Information is available free of charge on the ACS Publications website. Description of $\lambda$-potentials, effect of neglecting the intepolation of Lennard-Jones interactions, titration results for Asp and Glu within the single-site representation, a comparison of $\mathrm{p} K_{\mathrm{a}}$ values for HEWL obtained with various $\lambda$-dynamics-based constant $\mathrm{pH}$ methods, demonstration that charge interpolation requires a single evaluation of the electrostatic potential for both single- and multisite representations, a Mathematica notebook with instructions and routines to fit $V^{\mathrm{MM}} .{ }^{67}$ The fork of GROMACS 2021 with constant $\mathrm{pH}$ implemented 
as described here, is availble for download free of charge from https://gitlab.com/gromacsconstantph/constantph. In addition to the source code, also instructions on how to setup and perform MD simulations are available.

\section{References}

(1) Hollingsworth, S. A.; Dror, R. O. Molecular Dynamics Simulation for All. Neuron 2018, 99, 1129-1143.

(2) Groenhof, G.; Modi, V.; Morozov, D. Observe while it happens: catching photoactive proteins in the act with non-adiabatic molecular dynamics simulations. Curr. Opin. Struct. Biol. 2020, 61, 106-112.

(3) Warshel, A.; Sussman, F.; King, G. Free energy of charges in solvated proteins: microscopic calculations using a reversible charging process. Biochemistry 1986, 25, 83688372 .

(4) Alexov, E.; Mehler, E. L.; Baker, N.; M. Baptista, A.; Huang, Y.; Milletti, F.; Erik Nielsen, J.; Farrell, D.; Carstensen, T.; Olsson, M. H. M.; Shen, J. K.; Warwicker, J.; Williams, S.; Word, J. M. Progress in the prediction of pKa values in proteins. Proteins: Structure, Function, and Bioinformatics 2011, 79, 3260-3275.

(5) Chen, W.; Morrow, B. H.; Shi, C.; Shen, J. K. Recent development and application of constant pH molecular dynamics. Molecular simulation 2014, 40, 830-838.

(6) Baptista, A. M.; Martel, P. J.; Petersen, S. B. Simulation of protein conformational freedom as a function of $\mathrm{pH}$ : constant-pH molecular dynamics using implicit titration. Proteins: Structure, Function, and Bioinformatics 1997, 27, 523-544.

(7) Baptista, A. M.; Teixeira, V. H.; Soares, C. M. Constant-pH molecular dynamics using stochastic titration. The Journal of Chemical Physics 2002, 117, 4184-4200. 
(8) Bürgi, R.; Kollman, P. A.; van Gunsteren, W. F. Simulating proteins at constant pH: An approach combining molecular dynamics and Monte Carlo simulation. Proteins: Structure, Function, and Bioinformatics 2002, 47, 469-480.

(9) Mongan, J.; Case, D. A.; McCammon, J. A. Constant pH molecular dynamics in generalized Born implicit solvent. Journal of Computational Chemistry 2004, 25, 2038-2048.

(10) Meng, Y.; Roitberg, A. E. Constant pH Replica Exchange Molecular Dynamics in Biomolecules Using a Discrete Protonation Model. Journal of Chemical Theory and Computation 2010, 6, 1401-1412, PMID: 20514364.

(11) Itoh, S. G.; Damjanovic, A.; Brooks, B. R. pH replica-exchange method based on discrete protonation states. Proteins: Structure, Function, and Bioinformatics 2011, 79, 3420-3436.

(12) Swails, J. M.; York, D. M.; Roitberg, A. E. Constant pH Replica Exchange Molecular Dynamics in Explicit Solvent Using Discrete Protonation States: Implementation, Testing, and Validation. Journal of Chemical Theory and Computation 2014, 10, 13411352, PMID: 24803862.

(13) Börjesson, U.; Hünenberger, P. H. Explicit-solvent molecular dynamics simulation at constant pH: Methodology and application to small amines. The Journal of Chemical Physics 2001, 114, 9706-9719.

(14) Lee, M. S.; Salsbury Jr., F. R.; Brooks III, C. L. Constant-pH molecular dynamics using continuous titration coordinates. Proteins: Structure, Function, and Bioinformatics 2004, 56, 738-752.

(15) Khandogin, J.; III, C. L. B. Constant pH Molecular Dynamics with proton tautomerism. Biophys. J. 2005, 89, 141-157. 
(16) Donnini, S.; Tegeler, F.; Groenhof, G.; Grubmüller, H. Constant pH molecular dynamics in explicit solvent with $\lambda$-dynamics. Journal of chemical theory and computation $\mathbf{2 0 1 1}$, 7, 1962-1978.

(17) Wallace, J. A.; Shen, J. K. Continuous Constant pH Molecular Dynamics in Explicit Solvent with pH-Based Replica Exchange. Journal of chemical theory and computation 2011, ๆ, 2617-2629.

(18) Wallace, J. A.; Shen, J. K. Charge-leveling and proper treatment of long-range electrostatics in all-atom molecular dynamics at constant pH. J. Chem. Phys. 2012, 137, 184105.

(19) Goh, G. B.; Knight, J. L.; Brooks, C. L. Constant pH Molecular Dynamics Simulations of Nucleic Acids in Explicit Solvent. Journal of chemical theory and computation 2012, $8,36-46$.

(20) Chen, W.; Wallace, J. A.; Yue, Z.; Shen, J. K. Introducing Titratable Water to AllAtom Molecular Dynamics at Constant pH. Biophys. J. 2013, 105, L15-L17.

(21) Lee, J.; Miller, B. T.; Damjanovic, A.; Brooks, B. R. Constant pH Molecular Dynamics in Explicit Solvent with Enveloping Distribution Sampling and Hamiltonian Exchange. Journal of Chemical Theory and Computation 2014, 10, 2738-2750, PMID: 25061443.

(22) Dobrev, P.; Donnini, S.; Groenhof, G.; Grubmüller, H. Accurate Three States Model for Amino Acids with Two Chemically Coupled Titrating Sites in Explicit Solvent Atomistic Constant pH Simulations and p K a Calculations. Journal of chemical theory and computation 2017, 13, 147-160.

(23) Huang, Y.; Chen, W.; Wallace, J. A.; Shen, J. All-atom continuous constant pH molecular dynamics with particle mesh Ewald and titratable water. Journal of chemical theory and computation 2016, 12, 5411-5421. 
(24) Huang, Y.; Harris, R. C.; Shen, J. Generalized Born based continuous constant pH molecular dynamics in Amber: Implementation, benchmarking and analysis. Journal of chemical information and modeling 2018, 58, 1372-1383.

(25) Harris, R. C.; Shen, J. GPU-accelerated implementation of continuous constant pH molecular dynamics in amber: pKa predictions with single-pH simulations. Journal of chemical information and modeling 2019, 59, 4821-4832.

(26) Grünewald, F.; Souza, P. C.; Abdizadeh, H.; Barnoud, J.; de Vries, A. H.; Marrink, S. J. Titratable Martini model for constant $\mathrm{pH}$ simulations. The Journal of chemical physics 2020, 153, 024118.

(27) Mongan, J.; Case, D. A. Biomolecular simulations at constant pH. Current Opinion in Structural Biology 2005, 15, 157 - 163, Theory and simulation/Macromolecular assemblages.

(28) Damjanovic, A.; Miller, B. T.; Okur, A.; Brooks, B. R. Reservoir pH replica exchange. The Journal of Chemical Physics 2018, 149, 072321.

(29) Chen, Y.; Roux, B. Constant-pH Hybrid Nonequilibrium Molecular Dynamics - Monte Carlo Simulation Method. Journal of Chemical Theory and Computation 2015, 11, 3919-3931, PMID: 26300709.

(30) Kong, X.; Brooks III, C. L. $\lambda$-dynamics: A new approach to free energy calculations. The Journal of chemical physics 1996, 105, 2414-2423.

(31) Simonson, T.; Carlsson, J.; Case, D. A. Proton binding to proteins: p K a calculations with explicit and implicit solvent models. Journal of the American Chemical Society 2004, 126, 4167-4180.

(32) Chen, W.; Shen, J. K. Effects of System Net Charge and Electrostic Truncation on All-Atom Constant pH Molecular Dynamics. J. Comp. Chem. 2014, 35, 1986-1996. 
(33) Marrink, S. J.; Risselada, H. J.; Yefimov, S.; Tieleman, D. P.; De Vries, A. H. The MARTINI force field: coarse grained model for biomolecular simulations. The journal of physical chemistry B 2007, 111, 7812-7824.

(34) Donnini, S.; Ullmann, R. T.; Groenhof, G.; Grubmüller, H. Charge-neutral constant $\mathrm{pH}$ molecular dynamics simulations using a parsimonious proton buffer. Journal of chemical theory and computation 2016, 12, 1040-1051.

(35) Dobrev, P.; Vemulapalli, S. P. B.; Nath, N.; Griesinger, C.; Grubmüller, H. Probing the accuracy of explicit solvent constant $\mathrm{pH}$ molecular dynamics simulations for peptides. Journal of chemical theory and computation 2020, 16, 2561-2569.

(36) Darden, T.; York, D.; Pedersen, L. Particle mesh Ewald: An Nlog(N) method for Ewald sums in large systems. The Journal of chemical physics 1993, 98, 10089-10092.

(37) Essmann, U.; Perera, L.; Berkowitz, M. L.; Darden, T.; Lee, H.; Pedersen, L. G. A smooth particle mesh Ewald method. The Journal of chemical physics 1995, 103, 85778593.

(38) Knight, J. L.; Brooks III, C. L. Multisite $\lambda$ dynamics for simulated structure-activity relationship studies. Journal of chemical theory and computation 2011, 7, 2728-2739.

(39) Goh, G. B.; Hulbert, B. S.; Zhou, H.; Brooks III, C. L. Constant pH molecular dynamics of proteins in explicit solvent with proton tautomerism. Proteins: structure, function, and bioinformatics 2014, 82, 1319-1331.

(40) Abraham, M. J.; Murtola, T.; Schulz, R.; Páll, S.; Smith, J. C.; Hess, B.; Lindahl, E. GROMACS: High performance molecular simulations through multi-level parallelism from laptops to supercomputers. SoftwareX 2015, 1, 19-25.

(41) Singhal, A. K.; Chien, K. Y.; Wu, W. G.; Rule, G. S. Solution structure of cardiotoxin V from Naja naja atra. Biochemistry 1993, 32, 8036-8044. 
(42) Ramanadham, M.; Sieker, L.; Jensen, L. Refinement of triclinic lysozyme: II. The method of stereochemically restrained least squares. Acta Crystallographica Section B: Structural Science 1990, 46, 63-69.

(43) Sauguet, L.; Poitevin, F.; Murail, S.; Van Renterghem, C.; Moraga-Cid, G.; Malherbe, L.; Thompson, A. W.; Koehl, P.; Corringer, P.-J.; Baaden, M.; Delarue, M. Structural basis for ion permeation mechanism in pentameric ligand-gated ion channels. The EMBO journal 2013, 32, 728-741.

(44) Lee, T.-W.; Qasim Jr, M.; Laskowski Jr, M.; James, M. N. Structural insights into the non-additivity effects in the sequence-to-reactivity algorithm for serine peptidases and their inhibitors. Journal of molecular biology 2007, 367, 527-546.

(45) Huang, J.; MacKerell Jr, A. D. CHARMM36 all-atom additive protein force field: Validation based on comparison to NMR data. Journal of computational chemistry 2013, 34, 2135-2145.

(46) Buslaev, P.; Aho, N.; Jansen, A.; Bauer, P.; Groenhof, G.; Hess, B. Best practice in constant pH MD simulations: accuracy and sampling. 2022,

(47) Martini-website, General purpose coarse-grained force field. http://cgmartini.nl/ index.php/tools2/proteins-and-bilayers/204-martinize.

(48) Kaminski, G. A.; Friesner, R. A.; Tirado-Rives, J.; Jorgensen, W. L. Evaluation and reparametrization of the OPLS-AA force field for proteins via comparison with accurate quantum chemical calculations on peptides. The Journal of Physical Chemistry B 2001, $105,6474-6487$.

(49) Buck, M.; Bouguet-Bonnet, S.; Pastor, R. W.; MacKerell Jr, A. D. Importance of the CMAP correction to the CHARMM22 protein force field: dynamics of hen lysozyme. Biophysical journal 2006, 90, L36-L38. 
(50) Durell, S. R.; Brooks, B. R.; Ben-Naim, A. Solvent-induced forces between two hydrophilic groups. The Journal of Physical Chemistry 1994, 98, 2198-2202.

(51) Neria, E.; Fischer, S.; Karplus, M. Simulation of activation free energies in molecular systems. The Journal of chemical physics 1996, 105, 1902-1921.

(52) Yesylevskyy, S. O.; Schäfer, L. V.; Sengupta, D.; Marrink, S. J. Polarizable water model for the coarse-grained MARTINI force field. PLoS computational biology 2010, 6, e1000810.

(53) Berendsen, H. J.; Postma, J. P.; van Gunsteren, W. F.; Hermans, J. Intermolecular forces; Springer, 1981; pp 331-342.

(54) De Jong, D. H.; Baoukina, S.; Ingólfsson, H. I.; Marrink, S. J. Martini straight: Boosting performance using a shorter cutoff and GPUs. Computer Physics Communications 2016, 199, 1-7.

(55) Bussi, G.; Donadio, D.; Parrinello, M. Canonical sampling through velocity rescaling. The Journal of chemical physics 2007, 126, 014101.

(56) Parrinello, M.; Rahman, A. Polymorphic transitions in single crystals: A new molecular dynamics method. Journal of Applied physics 1981, 52, 7182-7190.

(57) Hess, B.; Bekker, H.; Berendsen, H. J.; Fraaije, J. G. LINCS: a linear constraint solver for molecular simulations. Journal of computational chemistry 1997, 18, 1463-1472.

(58) Miyamoto, S.; Kollman, P. A. Settle: An analytical version of the SHAKE and RATTLE algorithm for rigid water models. Journal of computational chemistry 1992, 13, 952962.

(59) Hub, J. S.; de Groot, B. L.; Grubmüller, H.; Groenhof, G. Quantifying artifacts in Ewald simulations of inhomogeneous systems with a net charge. Journal of chemical theory and computation 2014, 10, 381-390. 
(60) Thurlkill, R. L.; Grimsley, G. R.; Scholtz, J. M.; Pace, C. N. pK values of the ionizable groups of proteins. Protein science 2006, 15, 1214-1218.

(61) Tanokura, M. 1H-NMR study on the tautomerism of the imidazole ring of histidine residues: I. Microscopic pK values and molar ratios of tautomers in histidine-containing peptides. Biochimica et Biophysica Acta (BBA)-Protein Structure and Molecular Enzymology 1983, 742, 576-585.

(62) Chiang, C.-M.; Chien, K.-Y.; Lin, H.-j.; Lin, J.-F.; Yeh, H.-C.; Ho, P.-l.; Wu, W.-g. Conformational change and inactivation of membrane phospholipid-related activity of cardiotoxin V from Taiwan cobra venom at acidic pH. Biochemistry 1996, 35, 91679176.

(63) Chiang, C.-M.; Chang, S.-L.; Lin, H.-j.; Wu, W.-g. The role of acidic amino acid residues in the structural stability of snake cardiotoxins. Biochemistry 1996, 35, 9177-9186.

(64) Webb, H.; Tynan-Connolly, B. M.; Lee, G. M.; Farrell, D.; O’Meara, F.; Søndergaard, C. R.; Teilum, K.; Hewage, C.; McIntosh, L. P.; Nielsen, J. E. Remeasuring HEWL pKa values by NMR spectroscopy: Methods, analysis, accuracy, and implications for theoretical pKa calculations. Proteins: Structure, Function, and Bioinformatics 2011, 79, 685-702.

(65) Lee, J.; Miller, B. T.; Damjanovic, A.; Brooks, B. R. Enhancing constant-pH simulation in explicit solvent with a two-dimensional replica exchange method. Journal of chemical theory and computation 2015, 11, 2560-2574.

(66) Pezeshkian, W.; König, M.; Wassenaar, T. A.; Marrink, S. J. Backmapping triangulated surfaces to coarse-grained membrane models. Nature communications 2020, 11, 1-9.

(67) Wolfram Research, Inc., Mathematica, Version 11.3. Champaign, IL, 2018. 